JOURNAL OF THE

AMERICAN MATHEMATICAL SOCIETY

Volume 15, Number 2, Pages 497-529

S 0894-0347(01)00385-X

Article electronically published on December 28, 2001

\title{
CLUSTER ALGEBRAS I: FOUNDATIONS
}

\author{
SERGEY FOMIN AND ANDREI ZELEVINSKY
}

To the memory of Sergei Kerov

\section{INTRODUCTION}

In this paper, we initiate the study of a new class of algebras, which we call cluster algebras. Before giving precise definitions, we present some of the main features of these algebras. For any positive integer $n$, a cluster algebra $\mathcal{A}$ of rank $n$ is a commutative ring with unit and no zero divisors, equipped with a distinguished family of generators called cluster variables. The set of cluster variables is the (non-disjoint) union of a distinguished collection of $n$-subsets called clusters. These clusters have the following exchange property: for any cluster $\mathbf{x}$ and any element $x \in \mathbf{x}$, there is another cluster obtained from $\mathbf{x}$ by replacing $x$ with an element $x^{\prime}$ related to $x$ by a binomial exchange relation

$$
x x^{\prime}=M_{1}+M_{2},
$$

where $M_{1}$ and $M_{2}$ are two monomials without common divisors in the $n-1$ variables $\mathbf{x}-\{x\}$. Furthermore, any two clusters can be obtained from each other by a sequence of exchanges of this kind.

The prototypical example of a cluster algebra of rank 1 is the coordinate ring $\mathcal{A}=\mathbb{C}\left[S L_{2}\right]$ of the group $S L_{2}$, viewed in the following way. Writing a generic element of $S L_{2}$ as $\left[\begin{array}{ll}a & b \\ c & d\end{array}\right]$, we consider the entries $a$ and $d$ as cluster variables, and the entries $b$ and $c$ as scalars. There are just two clusters $\{a\}$ and $\{d\}$, and $\mathcal{A}$ is the algebra over the polynomial ring $\mathbb{C}[b, c]$ generated by the cluster variables $a$ and $d$ subject to the binomial exchange relation

$$
a d=1+b c .
$$

Another important incarnation of a cluster algebra of rank 1 is the coordinate ring $\mathcal{A}=\mathbb{C}\left[S L_{3} / N\right]$ of the base affine space of the special linear group $S L_{3}$; here $N$ is the maximal unipotent subgroup of $S L_{3}$ consisting of all unipotent upper triangular matrices. Using the standard notation $\left(x_{1}, x_{2}, x_{3}, x_{12}, x_{13}, x_{23}\right)$ for the Plücker coordinates on $S L_{3} / N$, we view $x_{2}$ and $x_{13}$ as cluster variables; then $\mathcal{A}$ is the algebra over the polynomial ring $\mathbb{C}\left[x_{1}, x_{3}, x_{12}, x_{13}\right]$ generated by the two cluster variables $x_{2}$ and $x_{13}$ subject to the binomial exchange relation

$$
x_{2} x_{13}=x_{1} x_{23}+x_{3} x_{12} .
$$

Received by the editors April 13, 2001 and, in revised form, October 26, 2001.

1991 Mathematics Subject Classification. Primary 14M99; Secondary $17 B 99$.

Key words and phrases. Cluster algebra, exchange pattern, Laurent phenomenon.

The authors were supported in part by NSF grants \#DMS-0049063, \#DMS-0070685 (S.F.), and \#DMS-9971362 (A.Z.). 
This form of representing the algebra $\mathbb{C}\left[S L_{3} / N\right]$ is closely related to the choice of a linear basis in it consisting of all monomials in the six Plücker coordinates which are not divisible by $x_{2} x_{13}$. This basis was introduced and studied in [9] under the name "canonical basis". As a representation of $S L_{3}$, the space $\mathbb{C}\left[S L_{3} / N\right]$ is the multiplicity-free direct sum of all irreducible finite-dimensional representations, and each of the components is spanned by a part of the above basis. Thus, this construction provides a "canonical" basis in every irreducible finite-dimensional representation of $S L_{3}$. After Lusztig's work [13, this basis had been recognized as (the classical limit at $q \rightarrow 1$ of) the dual canonical basis, i.e., the basis in the $q$-deformed algebra $\mathbb{C}_{q}\left[S L_{3} / N\right]$ which is dual to Lusztig's canonical basis in the appropriate $q$-deformed universal enveloping algebra (a.k.a. quantum group). The dual canonical basis in the space $\mathbb{C}[G / N]$ was later constructed explicitly for a few other classical groups $G$ of small rank: for $G=S p_{4}$ in [16] and for $G=S L_{4}$ in [2]. In both cases, $\mathbb{C}[G / N]$ can be seen to be a cluster algebra: there are 6 clusters of size 2 for $G=S p_{4}$, and 14 clusters of size 3 for $G=S L_{4}$.

We conjecture that the above examples can be extensively generalized: for any simply-connected connected semisimple group $G$, the coordinate rings $\mathbb{C}[G]$ and $\mathbb{C}[G / N]$, as well as coordinate rings of many other interesting varieties related to $G$, have a natural structure of a cluster algebra. This structure should serve as an algebraic framework for the study of "dual canonical bases" in these coordinate rings and their $q$-deformations. In particular, we conjecture that all monomials in the variables of any given cluster (the cluster monomials) belong to this dual canonical basis.

A particularly nice and well-understood example of a cluster algebra of an arbitrary rank $n$ is the homogeneous coordinate ring $\mathbb{C}\left[G r_{2, n+3}\right]$ of the Grassmannian of 2 -dimensional subspaces in $\mathbb{C}^{n+3}$. This ring is generated by the Plücker coordinates $[i j]$, for $1 \leq i<j \leq n+3$, subject to the relations

$$
[i k][j l]=[i j][k l]+[i l][j k],
$$

for all $i<j<k<l$. It is convenient to identify the indices $1, \ldots, n+3$ with the vertices of a convex $(n+3)$-gon, and the Plücker coordinates with its sides and diagonals. We view the sides $[12],[23], \ldots,[n+2, n+3],[1, n+3]$ as scalars, and the diagonals as cluster variables. The clusters are the maximal families of pairwise noncrossing diagonals; thus, they are in a natural bijection with the triangulations of this polygon. It is known that the cluster monomials form a linear basis in $\mathbb{C}\left[G r_{2, n+3}\right]$. To be more specific, we note that this ring is naturally identified with the ring of polynomial $S L_{2}$-invariants of an $(n+3)$-tuple of points in $\mathbb{C}^{2}$. Under this isomorphism, the basis of cluster monomials corresponds to the basis considered in [11, 18. (We are grateful to Bernd Sturmfels for bringing these references to our attention.)

An essential feature of the exchange relations (1.1) is that the right-hand side does not involve subtraction. Recursively applying these relations, one can represent any cluster variable as a subtraction-free rational expression in the variables of any given cluster. This positivity property is consistent with a remarkable connection between canonical bases and the theory of total positivity, discovered by G. Lusztig [14, 15]. Generalizing the classical concept of totally positive matrices, he defined totally positive elements in any reductive group $G$, and proved that all elements of the dual canonical basis in $\mathbb{C}[G]$ take positive values at them. 
It was realized in [15, 5] that the natural geometric framework for total positivity is given by double Bruhat cells, the intersections of cells of the Bruhat decompositions with respect to two opposite Borel subgroups. Different aspects of total positivity in double Bruhat cells were explored by the authors of the present paper and their collaborators in [1, 3, 4, 5, 6, 7, 12, 17, 20]. The binomial exchange relations of the form (1.1) played a crucial role in these studies. It was the desire to explain the ubiquity of these relations and to place them in a proper context that led us to the concept of cluster algebras. The crucial step in this direction was made in [20, where a family of clusters and exchange relations was explicitly constructed in the coordinate ring of an arbitrary double Bruhat cell. However, this family was not complete: in general, some clusters were missing, and not any member of a cluster could be exchanged from it. Thus, we started looking for a natural way to "propagate" exchange relations from one cluster to another. The concept of cluster algebras is the result of this investigation. We conjecture that the coordinate ring of any double Bruhat cell is a cluster algebra.

This article, in which we develop the foundations of the theory, is conceived as the first in a forthcoming series. We attempt to make the exposition elementary and self-contained; in particular, no knowledge of semisimple groups, quantum groups or total positivity is assumed on the part of the reader.

One of the main structural features of cluster algebras established in the present paper is the following Laurent phenomenon: any cluster variable $x$ viewed as a rational function in the variables of any given cluster is in fact a Laurent polynomial. This property is quite surprising: in most cases, the numerators of these Laurent polynomials contain a huge number of monomials, and the numerator for $x$ moves into the denominator when we compute the cluster variable $x^{\prime}$ obtained from $x$ by an exchange (1.1). The magic of the Laurent phenomenon is that, at every stage of this recursive process, a cancellation will inevitably occur, leaving a single monomial in the denominator.

In view of the positivity property discussed above, it is natural to expect that all Laurent polynomials for cluster variables will have positive coefficients. This seems to be a rather deep property; our present methods do not provide a proof of it.

On the bright side, it is possible to establish the Laurent phenomenon in many different situations spreading beyond the cluster algebra framework. One such extension is given in Theorem 3.2 By a modification of the method developed here, a large number of additional interesting instances of the Laurent phenomenon are established in a separate paper 8].

The paper is organized as follows. Section 2 contains an axiomatic definition, first examples and the first structural properties of cluster algebras. One of the technical difficulties in setting up the foundations involves the concept of an exchange graph whose vertices correspond to clusters, and the edges to exchanges among them. It is convenient to begin by taking the $n$-regular tree $\mathbb{T}_{n}$ as our underlying graph. This tree can be viewed as a universal cover for the actual exchange graph, whose appearance is postponed until Section 7

The Laurent phenomenon is established in Section 3 In Sections 4 and 5 , we scrutinize the main definition, obtain useful reformulations, and introduce some important classes of cluster algebras.

Section [6 contains a detailed analysis of cluster algebras of rank 2. This analysis exhibits deep and somewhat mysterious connections between cluster algebras and Kac-Moody algebras. This is just the tip of an iceberg: these connections will be 
further explored (for cluster algebras of an arbitrary rank) in the sequel to this paper. The main result of this sequel is a complete classification of cluster algebras of finite type, i.e., those with finitely many distinct clusters; cf. Example 7.6] This classification turns out to be yet another instance of the famous Cartan-Killing classification.

\section{MAIN DEFINITIONS}

Let $I$ be a finite set of size $n$; the standard choice will be $I=[n]=\{1,2, \ldots, n\}$. Let $\mathbb{T}_{n}$ denote the $n$-regular tree, whose edges are labeled by the elements of $I$, so that the $n$ edges emanating from each vertex receive different labels. By a common abuse of notation, we will sometimes denote by $\mathbb{T}_{n}$ the set of the tree's vertices. We will write $t \stackrel{i}{i} t^{\prime}$ if vertices $t, t^{\prime} \in \mathbb{T}_{n}$ are joined by an edge labeled by $i$.

To each vertex $t \in \mathbb{T}_{n}$, we will associate a cluster of $n$ generators ("variables") $\mathbf{x}(t)=\left(x_{i}(t)\right)_{i \in I}$. All these variables will commute with each other and satisfy the following exchange relations, for every edge $t \stackrel{j}{-} t^{\prime}$ in $\mathbb{T}_{n}$ :

$$
\begin{aligned}
& x_{i}(t)=x_{i}\left(t^{\prime}\right) \quad \text { for any } i \neq j ; \\
& x_{j}(t) x_{j}\left(t^{\prime}\right)=M_{j}(t)(\mathbf{x}(t))+M_{j}\left(t^{\prime}\right)\left(\mathbf{x}\left(t^{\prime}\right)\right) .
\end{aligned}
$$

Here $M_{j}(t)$ and $M_{j}\left(t^{\prime}\right)$ are two monomials in the $n$ variables $x_{i}, i \in I$; we think of these monomials as being associated with the two ends of the edge $t \stackrel{j}{-} t^{\prime}$.

To be more precise, let $\mathbb{P}$ be an abelian group without torsion, written multiplicatively. We call $\mathbb{P}$ the coefficient group; a prototypical example is a free abelian group of finite rank. Every monomial $M_{j}(t)$ in (2.2) will have the form

$$
M_{j}(t)=p_{j}(t) \prod_{i \in I} x_{i}^{b_{i}}
$$

for some coefficient $p_{j}(t) \in \mathbb{P}$ and some nonnegative integer exponents $b_{i}$.

The monomials $M_{j}(t)$ must satisfy certain conditions (axioms). To state them, we will need a little preparation. Let us write $P \mid Q$ to denote that a polynomial $P$ divides a polynomial $Q$. Accordingly, $x_{i} \mid M_{j}(t)$ means that the monomial $M_{j}(t)$ contains the variable $x_{i}$. For a rational function $F=F(x, y, \ldots)$, the notation $\left.F\right|_{x \leftarrow g(x, y, \ldots)}$ will denote the result of substituting $g(x, y, \ldots)$ for $x$ into $F$. To illustrate, if $F(x, y)=x y$, then $\left.F\right|_{x \leftarrow \frac{y}{x}}=\frac{y^{2}}{x}$.

Definition 2.1. An exchange pattern on $\mathbb{T}_{n}$ with coefficients in $\mathbb{P}$ is a family of monomials $\mathcal{M}=\left(M_{j}(t)\right)_{t \in \mathbb{T}_{n}, j \in I}$ of the form (2.3) satisfying the following four axioms:

If $t \in \mathbb{T}_{n}$, then $x_{j} \nmid M_{j}(t)$.

If $t_{1} \stackrel{j}{-} t_{2}$ and $x_{i} \mid M_{j}\left(t_{1}\right)$, then $x_{i} \nmid M_{j}\left(t_{2}\right)$.

If $t_{1} \stackrel{i}{-} t_{2} \stackrel{j}{=} t_{3}$, then $x_{j} \mid M_{i}\left(t_{1}\right)$ if and only if $x_{i} \mid M_{j}\left(t_{2}\right)$.

Let $t_{1} \stackrel{i}{-} t_{2} \stackrel{j}{-} t_{3} \stackrel{i}{-} t_{4}$. Then $\frac{M_{i}\left(t_{3}\right)}{M_{i}\left(t_{4}\right)}=\left.\frac{M_{i}\left(t_{2}\right)}{M_{i}\left(t_{1}\right)}\right|_{x_{j} \leftarrow M_{0} / x_{j}}$,

where $M_{0}=\left.\left(M_{j}\left(t_{2}\right)+M_{j}\left(t_{3}\right)\right)\right|_{x_{i}=0}$. 
We note that in the last axiom, the substitution $x_{j} \leftarrow \frac{M_{0}}{x_{j}}$ is effectively monomial, since in the event that neither $M_{j}\left(t_{2}\right)$ nor $M_{j}\left(t_{3}\right)$ contain $x_{i}$, condition (2.6) requires that both $M_{i}\left(t_{2}\right)$ and $M_{i}\left(t_{1}\right)$ do not depend on $x_{j}$, thus making the whole substitution irrelevant.

One easily checks that axiom (2.7) is invariant under the "flip" $t_{1} \leftrightarrow t_{4}, t_{2} \leftrightarrow t_{3}$, so no restrictions are added if we apply it "backwards". The axioms also imply at once that setting

$$
M_{j}^{\prime}(t)=M_{j}\left(t^{\prime}\right)
$$

for every edge $t \stackrel{j}{j} t^{\prime}$, we obtain another exchange pattern $\mathcal{M}^{\prime}$; this gives a natural involution $\mathcal{M} \rightarrow \mathcal{M}^{\prime}$ on the set of all exchange patterns.

Remark 2.2. Informally speaking, axiom (2.7) describes the propagation of an exchange pattern along the edges of $\mathbb{T}_{n}$. More precisely, let us fix the $2 n$ exchange monomials for all edges emanating from a given vertex $t$. This choice uniquely determines the ratio $M_{i}\left(t^{\prime}\right) / M_{i}\left(t^{\prime \prime}\right)$ for any vertex $t^{\prime}$ adjacent to $t$ and any edge $t^{\prime} \stackrel{i}{-} t^{\prime \prime}$ (to see this, take $t_{2}=t$ and $t_{3}=t^{\prime}$ in (2.7), and allow $i$ to vary). In view of (2.5), this ratio in turn uniquely determines the exponents of all variables $x_{k}$ in both monomials $M_{i}\left(t^{\prime}\right)$ and $M_{i}\left(t^{\prime \prime}\right)$. There remains, however, one degree of freedom in determining the coefficients $p_{i}\left(t^{\prime}\right)$ and $p_{i}\left(t^{\prime \prime}\right)$ because only their ratio is prescribed by (2.7). In Section 5 we shall introduce an important class of normalized exchange patterns for which this degree of freedom disappears, and so the whole pattern is uniquely determined by the $2 n$ monomials associated with edges emanating from a given vertex.

Let $\mathbb{Z} \mathbb{P}$ denote the group ring of $\mathbb{P}$ with integer coefficients. For an edge $t \stackrel{k}{t} t^{\prime}$, we refer to the binomial $P=M_{k}(t)+M_{k}\left(t^{\prime}\right) \in \mathbb{Z} \mathbb{P}\left[x_{i}: i \in I\right]$ as the exchange polynomial. We will write $t \frac{t^{\prime}}{t^{\prime}}$ or $t \frac{k}{P} t^{\prime}$ to indicate this fact. Note that, in view of the axiom (2.4), the right-hand side of the exchange relation (2.2) can be written as $P(\mathbf{x}(t))$, which is the same as $P\left(\mathbf{x}\left(t^{\prime}\right)\right)$.

Let $\mathcal{M}$ be an exchange pattern on $\mathbb{T}_{n}$ with coefficients in $\mathbb{P}$. Note that since $\mathbb{P}$ is torsion-free, the ring $\mathbb{Z} \mathbb{P}$ has no zero divisors. For every vertex $t \in \mathbb{T}_{n}$, let $\mathcal{F}(t)$ denote the field of rational functions in the cluster variables $x_{i}(t), i \in I$, with coefficients in $\mathbb{Z} \mathbb{P}$. For every edge $t \frac{j}{P} t^{\prime}$, we define a $\mathbb{Z P}$-linear field isomorphism $R_{t t^{\prime}}: \mathcal{F}\left(t^{\prime}\right) \rightarrow \mathcal{F}(t)$ by

$$
\begin{aligned}
& R_{t t^{\prime}}\left(x_{i}\left(t^{\prime}\right)\right)=x_{i}(t) \quad \text { for } i \neq k ; \\
& R_{t t^{\prime}}\left(x_{k}\left(t^{\prime}\right)\right)=\frac{P(\mathbf{x}(t))}{x_{k}(t)} .
\end{aligned}
$$

Note that property (2.4) ensures that $R_{t^{\prime} t}=R_{t t^{\prime}}^{-1}$. The transition maps $R_{t t^{\prime}}$ enable us to identify all the fields $\mathcal{F}(t)$ with each other. We can then view them as a single field $\mathcal{F}$ that contains all the elements $x_{i}(t)$, for all $t \in \mathbb{T}_{n}$ and $i \in I$. Inside $\mathcal{F}$, these elements satisfy the exchange relations (2.1) $-(2.2)$.

Definition 2.3. Let $\mathbb{A}$ be a subring with unit in $\mathbb{Z P}$ containing all coefficients $p_{i}(t)$ for $i \in I$ and $t \in \mathbb{T}_{n}$. The cluster algebra $\mathcal{A}=\mathcal{A}_{\mathbb{A}}(\mathcal{M})$ of rank $n$ over $\mathbb{A}$ associated 
with an exchange pattern $\mathcal{M}$ is the $\mathbb{A}$-subalgebra with unit in $\mathcal{F}$ generated by the union of all clusters $\mathbf{x}(t)$, for $t \in \mathbb{T}_{n}$.

The smallest possible ground ring $\mathbb{A}$ is the subring of $\mathbb{Z P}$ generated by all the coefficients $p_{i}(t)$; the largest one is $\mathbb{Z} \mathbb{P}$ itself. An intermediate choice of $\mathbb{A}$ appears in Proposition 2.6 below.

Since $\mathcal{A}$ is a subring of a field $\mathcal{F}$, it is a commutative ring with no zero divisors. We also note that if $\mathcal{M}^{\prime}$ is obtained from $\mathcal{M}$ by the involution (2.8), then the cluster algebra $\mathcal{A}_{\mathbb{A}}\left(\mathcal{M}^{\prime}\right)$ is naturally identified with $\mathcal{A}_{\mathbb{A}}(\mathcal{M})$.

Example 2.4. Let $n=1$. The tree $\mathbb{T}_{1}$ has only one edge $t \frac{1}{t} t^{\prime}$. The corresponding cluster algebra $\mathcal{A}$ has two generators $x=x_{1}(t)$ and $x^{\prime}=x_{1}\left(t^{\prime}\right)$ satisfying the exchange relation

$$
x x^{\prime}=p+p^{\prime},
$$

where $p$ and $p^{\prime}$ are arbitrary elements of the coefficient group $\mathbb{P}$. In the "universal" setting, we take $\mathbb{P}$ to be the free abelian group generated by $p$ and $p^{\prime}$. Then the two natural choices for the ground $\operatorname{ring} \mathbb{A}$ are the polynomial ring $\mathbb{Z}\left[p, p^{\prime}\right]$, and the Laurent polynomial ring $\mathbb{Z P}=\mathbb{Z}\left[p^{ \pm 1}, p^{\prime \pm 1}\right]$. All other realizations of $\mathcal{A}$ can be viewed as specializations of the universal one. Despite the seeming triviality of this example, it covers several important algebras: the coordinate ring of each of the varieties $S L_{2}, G r_{2,4}$ and $S L_{3} / B$ (cf. Section [1) is a cluster algebra of rank 1, for an appropriate choice of $\mathbb{P}, p, p^{\prime}$ and $\mathbb{A}$.

Example 2.5. Consider the case $n=2$. The tree $\mathbb{T}_{2}$ is shown below:

$$
\cdots \frac{1}{2} t_{0} \frac{2}{2} t_{1} \frac{1}{2} t_{2}-2-1
$$

Let us denote the cluster variables as follows:

$$
y_{1}=x_{1}\left(t_{0}\right)=x_{1}\left(t_{1}\right), \quad y_{2}=x_{2}\left(t_{1}\right)=x_{2}\left(t_{2}\right), \quad y_{3}=x_{1}\left(t_{2}\right)=x_{1}\left(t_{3}\right), \ldots
$$

(the above equalities among the cluster variables follow from (2.1)). Then the clusters look like

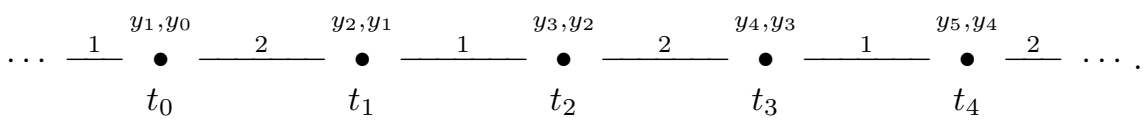

We claim that the exchange relations (2.2) can be written in the following form:

$$
\begin{array}{ll}
y_{0} y_{2}=q_{1} y_{1}^{b}+r_{1}, & y_{1} y_{3}=q_{2} y_{2}^{c}+r_{2}, \\
y_{2} y_{4}=q_{3} y_{3}^{b}+r_{3}, & y_{3} y_{5}=q_{4} y_{4}^{c}+r_{4}, \ldots,
\end{array}
$$

where the integers $b$ and $c$ are either both positive or both equal to 0 , and the coefficients $q_{m}$ and $r_{m}$ are elements of $\mathbb{P}$ satisfying the relations

$$
\begin{aligned}
q_{0} q_{2} r_{1}^{c} & =r_{0} r_{2}, & q_{1} q_{3} r_{2}^{b} & =r_{1} r_{3}, \\
q_{2} q_{4} r_{3}^{c} & =r_{2} r_{4}, & q_{3} q_{5} r_{4}^{b} & =r_{3} r_{5}, \ldots
\end{aligned}
$$

Furthermore, any such choice of parameters $b, c,\left(q_{m}\right),\left(r_{m}\right)$ results in a well-defined cluster algebra of rank 2 .

To prove this, we notice that, in view of (2.4) $-(2.5)$, both monomials $M_{2}\left(t_{0}\right)$ and $M_{2}\left(t_{1}\right)$ do not contain the variable $x_{2}$, and at most one of them contains $x_{1}$. If $x_{1}$ enters neither $M_{2}\left(t_{0}\right)$ nor $M_{2}\left(t_{1}\right)$, then these two are simply elements of $\mathbb{P}$. But 
then (2.6) forces all monomials $M_{i}\left(t_{m}\right)$ to be elements of $\mathbb{P}$, while (2.7) implies that it is possible to give the names $q_{m}$ and $r_{m}$ to the two monomials corresponding to each edge $t_{m}-t_{m+1}$ so that (2.11) -(2.12) hold with $b=c=0$.

Next, consider the case when precisely one of the monomials $M_{2}\left(t_{0}\right)$ and $M_{2}\left(t_{1}\right)$ contains $x_{1}$. Applying if necessary the involution (2.8) to our exchange pattern, we may assume that $M_{2}\left(t_{0}\right)=q_{1} x_{1}^{b}$ and $M_{2}\left(t_{1}\right)=r_{1}$ for some positive integer $b$ and some $q_{1}, r_{1} \in \mathbb{P}$. Thus, the exchange relation associated to the edge $t_{0} \stackrel{2}{\longrightarrow} t_{1}$ takes the form $y_{0} y_{2}=q_{1} y_{1}^{b}+r_{1}$. By (2.6) , we have $M_{1}\left(t_{1}\right)=q_{2} x_{2}^{c}$ and $M_{1}\left(t_{2}\right)=r_{2}$ for some positive integer $c$ and some $q_{2}, r_{2} \in \mathbb{P}$. Then the exchange relation for the edge $t_{1} \stackrel{1}{-} t_{2}$ takes the form $y_{1} y_{3}=q_{2} y_{2}^{c}+r_{2}$. At this point, we invoke (2.7):

$$
\frac{M_{2}\left(t_{2}\right)}{M_{2}\left(t_{3}\right)}=\left.\frac{M_{2}\left(t_{1}\right)}{M_{2}\left(t_{0}\right)}\right|_{x_{1} \leftarrow r_{2} / x_{1}}=\left.\frac{r_{1}}{q_{1} x_{1}^{b}}\right|_{x_{1} \leftarrow r_{2} / x_{1}}=\frac{r_{1} x_{1}^{b}}{q_{1} r_{2}^{b}} .
$$

By (2.5), we have $M_{2}\left(t_{2}\right)=q_{3} x_{1}^{b}$ and $M_{2}\left(t_{3}\right)=r_{3}$ for some $q_{3}, r_{3} \in \mathbb{P}$ satisfying $q_{1} q_{3} r_{2}^{b}=r_{1} r_{3}$. Continuing in the same way, we obtain all relations (2.11)-(2.12).

For fixed $b$ and $c$, the "universal" coefficient group $\mathbb{P}$ is the multiplicative abelian group generated by the elements $q_{m}$ and $r_{m}$ for all $m \in \mathbb{Z}$ subject to the defining relations (2.12). It is easy to see that this is a free abelian group of infinite rank. As a set of its free generators, one can choose any subset of $\left\{q_{m}, r_{m}: m \in \mathbb{Z}\right\}$ that contains four generators $q_{0}, r_{0}, q_{1}, r_{1}$ and precisely one generator from each pair $\left\{q_{m}, r_{m}\right\}$ for $m \neq 0,1$.

A nice specialization of this setup is provided by the homogeneous coordinate ring of the Grassmannian $G r_{2,5}$. Recall (cf. Section 1) that this ring is generated by the Plücker coordinates $[k, l]$, where $k$ and $l$ are distinct elements of the cyclic group $\mathbb{Z} / 5 \mathbb{Z}$. We shall write $\bar{m}=m \bmod 5 \in \mathbb{Z} / 5 \mathbb{Z}$ for $m \in \mathbb{Z}$, and adopt the convention $[k, l]=[l, k]$; see Figure 1

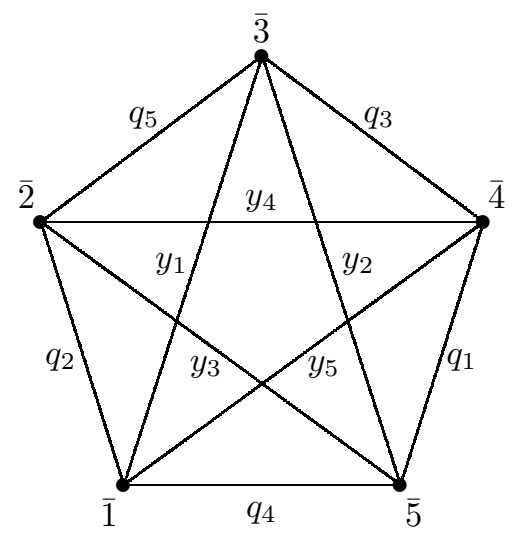

Figure 1. The Grassmannian $G r_{2,5}$

The ideal of relations among the Plücker coordinates is generated by the relations $[\bar{m}, \overline{m+2}][\overline{m+1}, \overline{m+3}]=[\bar{m}, \overline{m+1}][\overline{m+2}, \overline{m+3}]+[\bar{m}, \overline{m+3}][\overline{m+1}, \overline{m+2}]$

for $m \in \mathbb{Z}$. A direct check shows that these relations are a specialization of the relations (2.11), if we set $b=c=1, y_{m}=[\overline{2 m-1}, \overline{2 m+1}], q_{m}=[\overline{2 m-2}, \overline{2 m+2}]$, 
and $r_{m}=[\overline{2 m-2}, \overline{2 m-1}][\overline{2 m+1}, \overline{2 m+2}]=q_{m-2} q_{m+2}$ for all $m \in \mathbb{Z}$. The coefficient group $\mathbb{P}$ is the multiplicative free abelian group with 5 generators $q_{m}$. It is also immediate that the elements $q_{m}$ and $r_{m}$ defined in this way satisfy the relations (2.12).

We conclude this section by introducing two important operations on exchange patterns: restriction and direct product. Let us start with restriction. Let $\mathcal{M}$ be an exchange pattern of rank $n$ with an index set $I$ and coefficient group $\mathbb{P}$. Let $J$ be a subset of size $m$ in $I$. Let us remove from $\mathbb{T}_{n}$ all edges labeled by indices in $I-J$, and choose any connected component $\mathbb{T}$ of the resulting graph. This component is naturally identified with $\mathbb{T}_{m}$. Let $\mathcal{M}^{\prime}$ denote the restriction of $\mathcal{M}$ to $\mathbb{T}$, i.e., the collection of monomials $M_{j}(t)$ for all $j \in J$ and $t \in \mathbb{T}$. Then $\mathcal{M}^{\prime}$ is an exchange pattern on $\mathbb{T}$ whose coefficient group $\mathbb{P}^{\prime}$ is the direct product of $\mathbb{P}$ with the multiplicative free abelian group with generators $x_{i}, i \in I-J$. We shall say that $\mathcal{M}^{\prime}$ is obtained from $\mathcal{M}$ by restriction from $I$ to $J$. Note that $\mathcal{M}^{\prime}$ depends on the choice of a connected component $\mathbb{T}$, so there can be several different patterns obtained from $\mathcal{M}$ by restriction from $I$ to $J$. (We thank the anonymous referee for pointing this out.)

Proposition 2.6. Let $\mathcal{A}=\mathcal{A}_{\mathbb{A}}(\mathcal{M})$ be a cluster algebra of rank $n$ associated with an exchange pattern $\mathcal{M}$, and let $\mathcal{M}^{\prime}$ be obtained from $\mathcal{M}$ by restriction from $I$ to $J$ using a connected component $\mathbb{T}$. The $\mathbb{A}$-subalgebra of $\mathcal{A}$ generated by $\bigcup_{t \in \mathbb{T}} \mathbf{x}(t)$ is naturally identified with the cluster algebra $\mathcal{A}_{\mathbb{A}^{\prime}}\left(\mathcal{M}^{\prime}\right)$, where $\mathbb{A}^{\prime}$ is the polynomial $\operatorname{ring} \mathbb{A}\left[x_{i}: i \in I-J\right]$.

Proof. If $i \in I-J$, then (2.1) implies that $x_{i}(t)$ stays constant as $t$ varies over $\mathbb{T}$. Therefore, we can identify this variable with the corresponding generator $x_{i}$ of the coefficient group $\mathbb{P}^{\prime}$, and the statement follows.

Let us now consider two exchange patterns $\mathcal{M}_{1}$ and $\mathcal{M}_{2}$ of ranks $n_{1}$ and $n_{2}$, respectively, with index sets $I_{1}$ and $I_{2}$, and coefficient groups $\mathbb{P}_{1}$ and $\mathbb{P}_{2}$. We will construct the exchange pattern $\mathcal{M}=\mathcal{M}_{1} \times \mathcal{M}_{2}$ (the direct product of $\mathcal{M}_{1}$ and $\mathcal{M}_{2}$ ) of rank $n=n_{1}+n_{2}$, with the index set $I=I_{1} \sqcup I_{2}$, and coefficient group $\mathbb{P}=\mathbb{P}_{1} \times \mathbb{P}_{2}$. Consider the tree $\mathbb{T}_{n}$ whose edges are colored by $I$, and, for $\nu \in\{1,2\}$, let $\pi_{\nu}: \mathbb{T}_{n} \rightarrow \mathbb{T}_{n_{\nu}}$ be a map with the following property: if $t \frac{i}{\prime} t^{\prime}$ in $\mathbb{T}_{n}$ and $i \in I_{\nu}$ (resp., $i \in I-I_{\nu}=I_{3-\nu}$ ), then $\pi_{\nu}(t) \stackrel{i}{-} \pi_{\nu}\left(t^{\prime}\right)$ in $\mathbb{T}_{n_{\nu}}$ (resp., $\left.\pi_{\nu}(t)=\pi_{\nu}\left(t^{\prime}\right)\right)$. Clearly, such a map $\pi_{\nu}$ exists and is essentially unique: it is determined by specifying the image of any vertex of $\mathbb{T}_{n}$. We now introduce the exchange pattern $\mathcal{M}$ on $\mathbb{T}_{n}$ by setting, for every $t \in \mathbb{T}_{n}$ and $i \in I_{\nu} \subset I$, the monomial $M_{i}(t)$ to be equal to $M_{i}\left(\pi_{\nu}(t)\right)$, the latter monomial coming from the exchange pattern $\mathcal{M}_{\nu}$. The axioms (2.4) (2.7) for $\mathcal{M}$ are checked directly.

Proposition 2.7. Let $\mathcal{A}_{1}=\mathcal{A}_{\mathbb{A}_{1}}\left(\mathcal{M}_{1}\right)$ and $\mathcal{A}_{2}=\mathcal{A}_{\mathbb{A}_{2}}\left(\mathcal{M}_{2}\right)$ be cluster algebras. Let $\mathcal{M}=\mathcal{M}_{1} \times \mathcal{M}_{2}$ and $\mathbb{A}=\mathbb{A}_{1} \otimes \mathbb{A}_{2}$. Then the cluster algebra $\mathcal{A}_{\mathbb{A}}(\mathcal{M})$ is canonically isomorphic to the tensor product of algebras $\mathcal{A}_{1} \otimes \mathcal{A}_{2}$ (all tensor products are taken over $\mathbb{Z})$.

Proof. Let us identify each cluster variable $x_{i}(t)$, for $t \in \mathbb{T}_{n}$ and $i \in I_{1} \subset I$ (resp., $i \in I_{2} \subset I$ ), with $x_{i}\left(\pi_{1}(t)\right) \otimes 1$ (resp., $\left.1 \otimes x_{i}\left(\pi_{2}(t)\right)\right)$. Under this identification, the exchange relations for the exchange pattern $\mathcal{M}$ become identical to the exchange relations for $\mathcal{M}_{1}$ and $\mathcal{M}_{2}$. 


\section{THE LAURENT PHENOMENON}

In this section we prove the following important property of cluster algebras.

Theorem 3.1. In a cluster algebra, any cluster variable is expressed in terms of any given cluster as a Laurent polynomial with coefficients in the group ring $\mathbb{Z} \mathbb{P}$.

We conjecture that each of the coefficients in these Laurent polynomials is actually a nonnegative integer linear combination of elements in $\mathbb{P}$.

We will obtain Theorem 3.1 as a corollary of a more general result, which applies to more general underlying graphs and more general (not necessarily binomial) exchange polynomials.

Since Theorem 3.1 is trivial for $n=1$, we shall assume that $n \geq 2$. For every $m \geq 1$, let $\mathbb{T}_{n, m}$ be a tree of the form shown in Figure 2 The tree $\mathbb{T}_{n, m}$ has $m$ vertices of degree $n$ in its "spine" and $m(n-2)+2$ vertices of degree 1 . We label every edge of the tree by an element of an $n$-element index set $I$, so that the $n$ edges incident to each vertex on the spine receive different labels. (The reader may wish to think of the tree $\mathbb{T}_{n, m}$ as being part of the $n$-regular tree $\mathbb{T}_{n}$ of the cluster-algebra setup.)

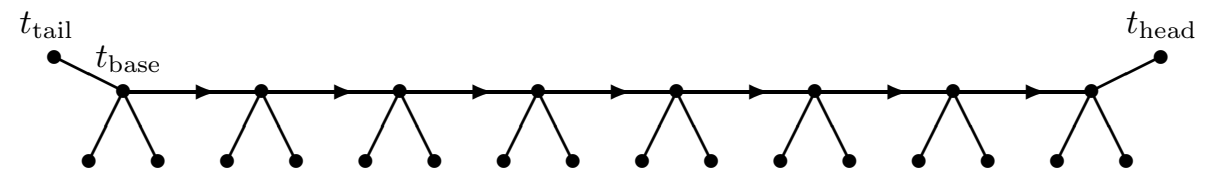

Figure 2. The "caterpillar" tree $\mathbb{T}_{n, m}$, for $n=4, m=8$

We fix two vertices $t_{\text {head }}$ and $t_{\text {tail }}$ of $\mathbb{T}_{n, m}$ that do not belong to the spine and are connected to its opposite ends. This gives rise to the orientation on the spine: away from $t_{\text {tail }}$ and towards $t_{\text {head }}$ (see Figure 2).

As before, let $\mathbb{P}$ be an abelian group without torsion, written multiplicatively. Let $\mathbb{Z}_{>0} \mathbb{P}$ denote the additive semigroup generated by $\mathbb{P}$ in the integer group ring $\mathbb{Z} \mathbb{P}$. Assume that a nonzero polynomial $P$ in the variables $x_{i}, i \in I$, with coefficients in $\mathbb{Z}_{\geq 0} \mathbb{P}$, is associated with every edge $t-t^{\prime}$ of $\mathbb{T}_{n, m}$. We call $P$ an exchange polynomial, and write $t \frac{}{P} t^{\prime}$ to describe this situation. Suppose that the exchange polynomials associated with the edges of $\mathbb{T}_{n, m}$ satisfy the following conditions:

(3.1) An exchange polynomial associated with an edge labeled by $j$ does not depend on $x_{j}$, and is not divisible by any $x_{i}, i \in I$.

) If $t_{0} \frac{i}{P} t_{1} \underset{Q}{\stackrel{j}{\longrightarrow}} t_{2} \frac{i}{R} t_{3}$, then $R=C \cdot\left(\left.P\right|_{x_{j} \leftarrow Q_{0} / x_{j}}\right)$, where $Q_{0}=\left.Q\right|_{x_{i}=0}$, and $C$ is a Laurent polynomial with coefficients in $\mathbb{Z}_{\geq 0} \mathbb{P}$.

(Note the orientation of the edge $t_{1} \rightarrow t_{2}$ in (3.2).)

For every vertex $t$ on the spine, let $\mathcal{P}(t)$ denote the family of $n$ exchange polynomials associated with the edges emanating from $t$. Also, let $\mathcal{C}$ denote the collection of all Laurent polynomials $C$ that appear in condition (3.2), for all possible choices 
of $t_{0}, t_{1}, t_{2}, t_{3}$, and let $\mathbb{A} \subset \mathbb{Z} \mathbb{P}$ denote the subring with unit generated by all coefficients of the Laurent polynomials from $\mathcal{P}\left(t_{\text {base }}\right) \cup \mathcal{C}$, where $t_{\text {base }}$ is the vertex on the spine connected with $t_{\text {tail }}$.

As before, we associate a cluster $\mathbf{x}(t)=\left\{x_{i}(t): i \in I\right\}$ to each vertex $t \in \mathbb{T}_{n, m}$, and consider the field $\mathcal{F}(t)$ of rational functions in these variables with coefficients in $\mathbb{Z} \mathbb{P}$. All these fields are identified with each other by the transition isomorphisms $R_{t t^{\prime}}: \mathcal{F}\left(t^{\prime}\right) \rightarrow \mathcal{F}(t)$ defined as in (2.9). We then view the fields $\mathcal{F}(t)$ as a single field $\mathcal{F}$ that contains all the elements $x_{i}(t)$, for $t \in \mathbb{T}_{n, m}$ and $i \in I$. These elements satisfy the exchange relations (2.1) and the following version of (2.2):

$$
x_{j}(t) x_{j}\left(t^{\prime}\right)=P(\mathbf{x}(t)),
$$

for any edge $t \frac{j}{P} t^{\prime}$ in $\mathbb{T}_{n, m}$.

Theorem 3.2. If conditions 3.1-3.2) are satisfied, then each element of the cluster $\mathbf{x}\left(t_{\text {head }}\right)$ is a Laurent polynomial in the cluster $\mathbf{x}\left(t_{\text {tail }}\right)$, with coefficients in the $\operatorname{ring} \mathbb{A}$.

We note that Theorem 3.2 is indeed a generalization of Theorem 3.1 for the following reasons:

- $\mathbb{T}_{n, m}$ is naturally embedded into $\mathbb{T}_{n}$;

- conditions (3.1)-3.2) are less restrictive than (2.4)-2.7)

- the claim being made in Theorem 3.2 about coefficients of the Laurent polynomials is stronger than that of Theorem 3.1 since $\mathbb{A} \subset \mathbb{Z} \mathbb{P}$.

Proof. We start with some preparations. We shall write any Laurent polynomial $L$ in the variables $\mathbf{x}=\left\{x_{i}: i \in I\right\}$ in the form

$$
L(\mathbf{x})=\sum_{\alpha \in S} u_{\alpha}(L) x^{\alpha},
$$

where all coefficients $u_{\alpha}(L)$ are nonzero, $S$ is a finite subset of the lattice $\mathbb{Z}^{I}$ (i.e., the lattice of rank $n$ with coordinates labeled by $I$ ), and $x^{\alpha}$ is the usual shorthand for $\prod_{i} x_{i}^{\alpha_{i}}$. The set $S$ is called the support of $L$ and denoted by $S=S(L)$.

Notice that once we fix the collection $\mathcal{C}$, condition 3.2 can be used as a recursive rule for computing $\mathcal{P}\left(t^{\prime}\right)$ from $\mathcal{P}(t)$, for any edge $t \stackrel{j}{\longrightarrow} t^{\prime}$ on the spine. It follows that the whole pattern of exchange polynomials is determined by the families of polynomials $\mathcal{P}\left(t_{\text {base }}\right)$ and $\mathcal{C}$. Moreover, since these polynomials have coefficients in $\mathbb{Z}_{>0} \mathbb{P}$, and the expression for $R$ in (3.2) does not involve subtraction, it follows that the support of any exchange polynomial is uniquely determined by the supports of the polynomials from $\mathcal{P}\left(t_{\text {base }}\right)$ and $\mathcal{C}$. Note that condition (3.1) can be formulated as a set of restrictions on these supports. In particular, it requires that in the situation of (3.2), the Laurent polynomial $C$ does not depend on $x_{i}$ and is a polynomial in $x_{j}$; in other words, every $\alpha \in S(C)$ should have $\alpha_{i}=0$ and $\alpha_{j} \geq 0$.

We now fix a family of supports $S(L)$, for all $L \in \mathcal{P}\left(t_{\text {base }}\right) \cup \mathcal{C}$, and assume that this family complies with (3.1). As is common in algebra, we shall view the coefficients $u_{\alpha}(L)$, for all $L \in \mathcal{P}\left(t_{\text {base }}\right) \cup \mathcal{C}$ and $\alpha \in S(L)$, as indeterminates. Then all the coefficients in all exchange polynomials become "canonical" (i.e., independent of the choice of $\mathbb{P}$ ) polynomials in these indeterminates, with positive integer coefficients. 
The above discussion shows that it suffices to prove our theorem in the following "universal coefficients" setup: let $\mathbb{P}$ be the free abelian group (written multiplicatively) with generators $u_{\alpha}(L)$, for all $L \in \mathcal{P}\left(t_{\text {base }}\right) \cup \mathcal{C}$ and $\alpha \in S(L)$. Under this assumption, $\mathbb{A}$ is simply the integer polynomial ring in the indeterminates $u_{\alpha}(L)$.

Recall that we can view all cluster variables $x_{i}(t)$ as elements of the field $\mathcal{F}\left(t_{\text {tail }}\right)$ of rational functions in the cluster $x\left(t_{\text {tail }}\right)$ with coefficients in $\mathbb{Z P}$. For $t \in \mathbb{T}_{n, m}$, let $\mathcal{L}(t)$ denote the ring of Laurent polynomials in the cluster $\mathbf{x}(t)$, with coefficients in $\mathbb{A}$. We view each $\mathcal{L}(t)$ as a subring of the ambient field $\mathcal{F}\left(t_{\text {tail }}\right)$.

In this terminology, our goal is to show that the cluster $\mathbf{x}\left(t_{\text {head }}\right)$ is contained in $\mathcal{L}\left(t_{\text {tail }}\right)$. We proceed by induction on $m$, the size of the spine. The claim is trivial for $m=1$, so let us assume that $m \geq 2$, and furthermore assume that our statement is true for all "caterpillars" with smaller spine.

Let us abbreviate $t_{0}=t_{\text {tail }}$ and $t_{1}=t_{\text {base }}$, and suppose that the path from $t_{\text {tail }}$ to $t_{\text {head }}$ starts with the following two edges: $t_{0} \frac{i}{P} t_{1} \frac{j}{Q} t_{2}$. Let $t_{3} \in \mathbb{T}_{n, m}$ be the vertex such that $t_{2} \frac{i}{R} t_{3}$.

The following lemma plays a crucial role in our proof.

Lemma 3.3. The clusters $\mathbf{x}\left(t_{1}\right), \mathbf{x}\left(t_{2}\right)$, and $\mathbf{x}\left(t_{3}\right)$ are contained in $\mathcal{L}\left(t_{0}\right)$. Furthermore, $\operatorname{gcd}\left(x_{i}\left(t_{3}\right), x_{i}\left(t_{1}\right)\right)=\operatorname{gcd}\left(x_{j}\left(t_{2}\right), x_{i}\left(t_{1}\right)\right)=1$ (as elements of $\left.\mathcal{L}\left(t_{0}\right)\right)$.

Note that $\mathcal{L}_{0}=\mathcal{L}\left(t_{0}\right)$ is a unique factorization domain, so any two elements $x, y \in \mathcal{L}_{0}$ have a well-defined greatest common divisor $\operatorname{gcd}(x, y)$, which is an element of $\mathcal{L}_{0}$ defined up to a multiple from the group $\mathcal{L}_{0}^{\times}$of units (that is, invertible elements) of $\mathcal{L}_{0}$. In our "universal" situation, $\mathcal{L}_{0}^{\times}$consists of Laurent monomials in the cluster $\mathbf{x}\left(t_{0}\right)$ with coefficients \pm 1 .

Proof. The only element from the clusters $\mathbf{x}\left(t_{1}\right), \mathbf{x}\left(t_{2}\right)$, and $\mathbf{x}\left(t_{3}\right)$ whose inclusion in $\mathcal{L}_{0}$ is not immediately obvious is $x_{i}\left(t_{3}\right)$. To simplify the notation, let us denote $x=x_{i}\left(t_{0}\right), y=x_{j}\left(t_{0}\right)=x_{j}\left(t_{1}\right), z=x_{i}\left(t_{1}\right)=x_{i}\left(t_{2}\right), u=x_{j}\left(t_{2}\right)=x_{j}\left(t_{3}\right)$, and $v=x_{i}\left(t_{3}\right)$, so that these variables appear in the clusters at $t_{0}, \ldots, t_{3}$, as shown below:

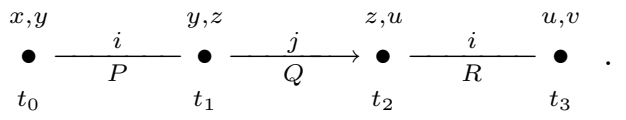

Note that the variables $x_{k}$, for $k \notin\{i, j\}$, do not change as we move among the four clusters under consideration. The lemma is then restated as saying that

$$
\begin{aligned}
& v \in \mathcal{L}_{0} ; \\
& \operatorname{gcd}(z, u)=1\left(\text { as elements of } \mathcal{L}_{0}\right) ; \\
& \operatorname{gcd}(z, v)=1\left(\text { as elements of } \mathcal{L}_{0}\right) .
\end{aligned}
$$

Another notational convention will be based on the fact that each of the polynomials $P, Q, R$ has a distinguished variable on which it depends, namely $x_{j}$ for $P$ and $R$, and $x_{i}$ for $Q$. (In view of (3.1), $P$ and $R$ do not depend on $x_{i}$, while $Q$ does not depend on $x_{j}$.) With this in mind, we will routinely write $P, Q$, and $R$ as polynomials in one (distinguished) variable. In the same spirit, the notation $Q^{\prime}$, $R^{\prime}$, etc., will refer to the partial derivative with respect to the distinguished variable.

We will prove the statements (3.3), (3.4), and (3.5) one by one, in this order. 
By (3.2), the polynomial $R$ is given by

$$
R(u)=C(u) P\left(\frac{Q(0)}{u}\right),
$$

where $C$ is an "honest" polynomial in $u$ and a Laurent polynomial in the "mute" variables $x_{k}, k \notin\{i, j\}$. (Recall that $C$ does not depend on $x_{i}$.) We then have:

$$
\begin{aligned}
& z=\frac{P(y)}{x} ; \\
& u=\frac{Q(z)}{y}=\frac{Q\left(\frac{P(y)}{x}\right)}{y} ; \\
& v=\frac{R(u)}{z}=\frac{R\left(\frac{Q(z)}{y}\right)}{z}=\frac{R\left(\frac{Q(z)}{y}\right)-R\left(\frac{Q(0)}{y}\right)}{z}+\frac{R\left(\frac{Q(0)}{y}\right)}{z} .
\end{aligned}
$$

Since

$$
\frac{R\left(\frac{Q(z)}{y}\right)-R\left(\frac{Q(0)}{y}\right)}{z} \in \mathcal{L}_{0}
$$

and

$$
\frac{R\left(\frac{Q(0)}{y}\right)}{z}=\frac{C\left(\frac{Q(0)}{y}\right) P(y)}{z}=C\left(\frac{Q(0)}{y}\right) x \in \mathcal{L}_{0}
$$

(3.3) follows.

We next prove (3.4). We have

$$
u=\frac{Q(z)}{y} \equiv \frac{Q(0)}{y} \bmod z .
$$

Since $x$ and $y$ are invertible in $\mathcal{L}_{0}$, we conclude that $\operatorname{gcd}(z, u)=\operatorname{gcd}(P(y), Q(0))$. Now the trouble that we took in passing to universal coefficients finally pays off: since $P(y)$ and $Q(0)$ are nonzero polynomials in the cluster $\mathbf{x}\left(t_{0}\right)$ whose coefficients are distinct generators of the polynomial ring $\mathbb{A}$, it follows that $\operatorname{gcd}(P(y), Q(0))=1$, proving (3.4).

It remains to prove (3.5). Let

$$
f(z)=R\left(\frac{Q(z)}{y}\right) .
$$

Then

$$
v=\frac{f(z)-f(0)}{z}+C\left(\frac{Q(0)}{y}\right) x .
$$

Our goal is to show that $\operatorname{gcd}(z, v)=1$; to this end, we are going to compute $v \bmod z$ as "explicitly" as possible. We have, $\bmod z$,

$$
\frac{f(z)-f(0)}{z} \equiv f^{\prime}(0)=R^{\prime}\left(\frac{Q(0)}{y}\right) \cdot \frac{Q^{\prime}(0)}{y} .
$$

Hence

$$
v \equiv R^{\prime}\left(\frac{Q(0)}{y}\right) \cdot \frac{Q^{\prime}(0)}{y}+C\left(\frac{Q(0)}{y}\right) x \bmod z .
$$

Note that the right-hand side is a linear polynomial in $x$, whose coefficients are Laurent polynomials in the rest of the variables of the cluster $\mathbf{x}\left(t_{0}\right)$. Thus our claim will follow if we show that $\operatorname{gcd}\left(C\left(\frac{Q(0)}{y}\right), P(y)\right)=1$. This, again, is a 
consequence of our "universal coefficients" setup since the coefficients of $C, P$ and $Q$ are distinct generators of the polynomial ring $\mathbb{A}$.

We can now complete the proof of Theorem 3.2. We need to show that any variable $x=x_{k}\left(t_{\text {head }}\right)$ belongs to $\mathcal{L}\left(t_{0}\right)$. Since both $t_{1}$ and $t_{3}$ are closer to $t_{\text {head }}$ than $t_{0}$, we can use the inductive assumption to conclude that $x$ belongs to both $\mathcal{L}\left(t_{1}\right)$ and $\mathcal{L}\left(t_{3}\right)$. Since $x \in \mathcal{L}\left(t_{1}\right)$, it follows from (2.1) that $x$ can be written as $x=f / x_{i}\left(t_{1}\right)^{a}$ for some $f \in \mathcal{L}\left(t_{0}\right)$ and $a \in \mathbb{Z}_{\geq 0}$. On the other hand, since $x \in \mathcal{L}\left(t_{3}\right)$, it follows from (2.1) and from the inclusion $x_{i}\left(t_{3}\right) \in \mathcal{L}\left(t_{0}\right)$ guaranteed by Lemma 3.3 that $x$ has the form $x=g / x_{j}\left(t_{2}\right)^{b} x_{i}\left(t_{3}\right)^{c}$ for some $g \in \mathcal{L}\left(t_{0}\right)$ and some $b, c \in \mathbb{Z}_{\geq 0}$. The inclusion $x \in \mathcal{L}\left(t_{0}\right)$ now follows from the fact that, by the last statement in Lemma 3.3 the denominators in the two obtained expressions for $x$ are coprime in $\mathcal{L}\left(t_{0}\right)$.

Several examples that can be viewed as applications of Theorem 3.2 are given in 8 .

\section{EXChange Relations: The exponents}

Let $\mathcal{M}=\left(M_{j}(t)\right): t \in \mathbb{T}_{n}, j \in I$ ) be an exchange pattern (see Definition 2.1). In this section we will ignore the coefficients in the monomials $M_{j}(t)$ and take a closer look at the dynamics of their exponents. (An alternative point of view that the reader may find helpful is to assume that all exchange patterns considered in this section will have all their coefficients $p_{j}(t)$ equal to 1.) For every edge $t \stackrel{j}{j} t^{\prime}$ in $\mathbb{T}_{n}$, let us write the ratio $M_{j}(t) / M_{j}\left(t^{\prime}\right)$ of the corresponding monomials as

$$
\frac{M_{j}(t)}{M_{j}\left(t^{\prime}\right)}=\frac{p_{j}(t)}{p_{j}\left(t^{\prime}\right)} \prod_{i} x_{i}^{b_{i j}(t)}
$$

where $b_{i j}(t) \in \mathbb{Z}$ (cf. (2.3) ); we note that ratios of this kind have already appeared in (2.7). Let us denote by $B(t)=\left(b_{i j}(t)\right)$ the $n \times n$ integer matrix whose entries are the exponents in (4.1). In view of (2.5), the exponents in $M_{j}(t)$ and $M_{j}\left(t^{\prime}\right)$ are recovered from $B(t)$ :

$$
\begin{aligned}
& M_{j}(t)=p_{j}(t) \prod_{i: b_{i j}(t)>0} x_{i}^{b_{i j}(t)}, \\
& M_{j}\left(t^{\prime}\right)=p_{j}\left(t^{\prime}\right) \prod_{i: b_{i j}(t)<0} x_{i}^{-b_{i j}(t)} .
\end{aligned}
$$

Thus, the family of matrices $(B(t))_{t \in \mathbb{T}_{n}}$ encodes all the exponents in all monomials of an exchange pattern.

We shall describe the conditions on the family of matrices $(B(t))$ imposed by the axioms of an exchange pattern. To do this, we need some preparation.

Definition 4.1. A square integer matrix $B=\left(b_{i j}\right)$ is called sign-skew-symmetric if, for any $i$ and $j$, either $b_{i j}=b_{j i}=0$, or else $b_{i j}$ and $b_{j i}$ are of opposite sign; in particular, $b_{i i}=0$ for all $i$.

Definition 4.2. Let $B=\left(b_{i j}\right)$ and $B^{\prime}=\left(b_{i j}^{\prime}\right)$ be square integer matrices of the same size. We say that $B^{\prime}$ is obtained from $B$ by the matrix mutation in direction $k$ 
and write $B^{\prime}=\mu_{k}(B)$ if

$$
b_{i j}^{\prime}= \begin{cases}-b_{i j} & \text { if } i=k \text { or } j=k ; \\ b_{i j}+\frac{\left|b_{i k}\right| b_{k j}+b_{i k}\left|b_{k j}\right|}{2} & \text { otherwise. }\end{cases}
$$

An immediate check shows that $\mu_{k}$ is involutive, i.e., its square is the identity transformation.

Proposition 4.3. A family of $n \times n$ integer matrices $(B(t))_{t \in \mathbb{T}_{n}}$ corresponds to an exchange pattern if and only if the following conditions hold:

(1) $B(t)$ is sign-skew-symmetric for any $t \in \mathbb{T}_{n}$.

(2) If $t \stackrel{k}{-} t^{\prime}$, then $B\left(t^{\prime}\right)=\mu_{k}(B(t))$.

Proof. We start with the "only if" part, i.e., we assume that the matrices $B(t)$ are determined by an exchange pattern via (4.1) and check the conditions (1)-(2). The condition $b_{j j}(t)=0$ follows from (2.4). The remaining part of (1) (dealing with $i \neq j$ ), follows at once from (2.6). Turning to part (2), the equality $b_{i k}^{\prime}=-b_{i k}$ is immediate from the definition (4.1). Now suppose that $j \neq k$. In this case, we apply the axiom (2.7) to the edge $t \frac{k}{2} t^{\prime}$ taken together with the two adjacent edges emanating from $t$ and $t^{\prime}$ and labeled by $j$. Taking (4.2) into account, we obtain:

$$
\prod_{i} x_{i}^{b_{i j}^{\prime}}=\left.\prod_{i} x_{i}^{b_{i j}}\right|_{x_{k} \leftarrow M / x_{k}}
$$

where $M=\prod_{i: b_{i k} b_{j k}<0} x_{i}^{\left|b_{i k}\right|}$. Comparing the exponents of $x_{k}$ on both sides of (4.4) yields $b_{k j}^{\prime}=-b_{k j}$. Finally, if $i \neq k$, then comparing the exponents of $x_{i}$ on both sides of (4.4) gives

$$
b_{i j}^{\prime}= \begin{cases}b_{i j} & \text { if } b_{i k} b_{j k} \geq 0 \\ b_{i j}+\left|b_{i k}\right| b_{k j} & \text { otherwise. }\end{cases}
$$

To complete the proof of (2), it remains to notice that, in view of the already proven part (1), the condition $b_{i k} b_{j k} \geq 0$ is equivalent to $b_{i k} b_{k j} \leq 0$, which makes the last formula equivalent to (4.3).

To prove the "if" part, it suffices to show that if the matrices $B(t)$ satisfy (1)(2), then the monomials $M_{j}(t)$ given by the first equality in 4.2) (with $p_{j}(t)=1$ ) satisfy the axioms of an exchange pattern. This is done by a direct check.

Since all matrix mutations are involutive, any choice of an initial vertex $t_{0} \in \mathbb{T}_{n}$ and an arbitrary $n \times n$ integer matrix $B$ gives rise to a unique family of integer matrices $B(t)$ satisfying condition (2) in Proposition 4.3 and such that $B\left(t_{0}\right)=B$. Thus, the exponents in all monomials $M_{j}(t)$ are uniquely determined by a single matrix $B=B\left(t_{0}\right)$. By Proposition 4.3 in order to determine an exchange pattern, $B$ must be such that all matrices obtained from it by a sequence of matrix mutations are sign-skew-symmetric. Verifying that a given matrix $B$ has this property seems to be quite nontrivial in general. Fortunately, there is another restriction on $B$ that is much easier to check, which implies the desired property, and still leaves us with a large class of matrices sufficient for most applications. 
Definition 4.4. A square integer matrix $B=\left(b_{i j}\right)$ is called skew-symmetrizable if there exists a diagonal skew-symmetrizing matrix $D$ with positive integer diagonal entries $d_{i}$ such that $D B$ is skew-symmetric, i.e., $d_{i} b_{i j}=-d_{j} b_{j i}$ for all $i$ and $j$.

Proposition 4.5. For every choice of a vertex $t_{0} \in \mathbb{T}_{n}$ and a skew-symmetrizable matrix $B$, there exists a unique family of matrices $(B(t))_{t \in \mathbb{T}_{n}}$ associated with an exchange pattern on $\mathbb{T}_{n}$ and such that $B\left(t_{0}\right)=B$. Furthermore, all the matrices $B(t)$ are skew-symmetrizable, sharing the same skew-symmetrizing matrix.

Proof. The proof follows at once from the following two observations:

1. Every skew-symmetrizable matrix $B$ is sign-skew-symmetric.

2. If $B$ is skew-symmetrizable and $B^{\prime}=\mu_{k}(B)$, then $B^{\prime}$ is also skew-symmetrizable, with the same skew-symmetrizing matrix.

We call an exchange pattern - and the corresponding cluster algebra-skewsymmetrizable if all the matrices $B(t)$ given by (4.1) (equivalently, one of them) are skew-symmetrizable. In particular, all cluster algebras of rank $n \leq 2$ are skewsymmetrizable: for $n=1$ we have $B(t) \equiv(0)$, while for $n=2$, the calculations in Example 2.5] show that one can take

$$
B\left(t_{m}\right)=(-1)^{m}\left[\begin{array}{cc}
0 & b \\
-c & 0
\end{array}\right]
$$

for all $m \in \mathbb{Z}$, in the notation of (2.10)-(2.11).

Remark 4.6. Skew-symmetrizable matrices are closely related to symmetrizable (generalized) Cartan matrices appearing in the theory of Kac-Moody algebras. More generally, to every sign-skew-symmetric matrix $B=\left(b_{i j}\right)$ we can associate a generalized Cartan matrix $A=A(B)=\left(a_{i j}\right)$ of the same size by setting

$$
a_{i j}= \begin{cases}2 & \text { if } i=j ; \\ -\left|b_{i j}\right| & \text { if } i \neq j .\end{cases}
$$

There seem to be deep connections between the cluster algebra corresponding to $B$ and the Kac-Moody algebra associated with $A(B)$. We exhibit such a connection for the rank 2 case in Section 6 below. This is however just the tip of an iceberg: a much more detailed analysis will be presented in the sequel to this paper.

In order to show that non-skew-symmetrizable exchange patterns do exist, we conclude this section by exhibiting a 3-parameter family of such patterns of rank 3 .

Proposition 4.7. Let $\alpha, \beta$, and $\gamma$ be three positive integers such that $\alpha \beta \gamma \geq 3$. There exists a unique family of matrices $(B(t))_{t \in \mathbb{T}_{3}}$ associated with a non-skewsymmetrizable exchange pattern on $\mathbb{T}_{3}$ and such that the matrix $B\left(t_{0}\right)$ at a given vertex $t_{0} \in \mathbb{T}_{3}$ is equal to

$$
B(\alpha, \beta, \gamma)=\left[\begin{array}{ccc}
0 & 2 \alpha & -2 \alpha \beta \\
-\beta \gamma & 0 & 2 \beta \\
\gamma & -\alpha \gamma & 0
\end{array}\right]
$$

Proof. First of all, the matrix $B(\alpha, \beta, \gamma)$ is sign-skew-symmetric but not skewsymmetrizable. Indeed, any skew-symmetrizable matrix $B=\left(b_{i j}\right)$ satisfies the equation $b_{12} b_{23} b_{31}=-b_{21} b_{32} b_{13}$. However, this equation for $B(\alpha, \beta, \gamma)$ holds only when $\alpha \beta \gamma$ is equal to 0 or 2 . 
For the purpose of this proof only, we refer to a $3 \times 3$ matrix $B$ as cyclical if its entries follow one of the two sign patterns

$$
\left[\begin{array}{ccc}
0 & + & - \\
- & 0 & + \\
+ & - & 0
\end{array}\right],\left[\begin{array}{ccc}
0 & - & + \\
+ & 0 & - \\
- & + & 0
\end{array}\right] .
$$

In particular, $B(\alpha, \beta, \gamma)$ is cyclical; to prove the proposition, it suffices to show that any matrix obtained from it by a sequence of matrix mutations is also cyclical.

The set of cyclical matrices is not stable under matrix mutations. Let us define some subsets of cyclical matrices that behave nicely with respect to matrix mutations. For a $3 \times 3$ matrix $B$, we denote

$$
c_{1}=\left|b_{23} b_{32}\right|, c_{2}=\left|b_{13} b_{31}\right|, c_{3}=\left|b_{12} b_{21}\right|, r=\left|b_{12} b_{23} b_{31}\right| \text {. }
$$

For $i \in\{1,2,3\}$, we say that $B$ is $i$-biased if we have

$$
r>c_{i} \geq r / 2 \geq c_{j} \geq 6
$$

for any $j \in\{1,2,3\} \backslash\{i\}$. For $B(\alpha, \beta, \gamma)$, we have

$$
r / 2=c_{1}=c_{2}=c_{3}=2 \alpha \beta \gamma \geq 6,
$$

so it is $i$-biased for every $i \in\{1,2,3\}$.

Our proposition becomes an immediate consequence of the following lemma.

Lemma 4.8. Suppose $B$ is cyclical and $i$-biased, and let $j \neq i$. Then $\mu_{j}(B)$ is cyclical and $j$-biased.

Proof. Without loss of generality we can assume that $i=1$ and $j=2$. Denote $B^{\prime}=\mu_{2}(B)$, and let us write $r^{\prime}=\left|b_{12}^{\prime} b_{23}^{\prime} b_{31}^{\prime}\right|, c_{1}^{\prime}=\left|b_{23}^{\prime} b_{32}^{\prime}\right|$, etc. By (4.3), we have $b_{12}^{\prime}=-b_{12}, b_{21}^{\prime}=-b_{21}, b_{23}^{\prime}=-b_{23}, b_{32}^{\prime}=-b_{32}$; therefore, $c_{1}^{\prime}=c_{1}$ and $c_{3}^{\prime}=c_{3}$. We also have

$$
b_{13}^{\prime}=b_{13}+\frac{\left|b_{12}\right| b_{23}+b_{12}\left|b_{23}\right|}{2}, b_{31}^{\prime}=b_{31}+\frac{\left|b_{32}\right| b_{21}+b_{32}\left|b_{21}\right|}{2} .
$$

Since $B$ is cyclical, the two summands on the right-hand side of each of the equalities in (4.8) have opposite signs. Note that

$$
\left|b_{12} b_{23}\right|=\frac{r}{\left|b_{31}\right|} \geq \frac{2 c_{2}}{\left|b_{31}\right|}=2\left|b_{13}\right|
$$

and

$$
\left|b_{32} b_{21}\right|=\frac{c_{1}\left|b_{21}\right|}{\left|b_{23}\right|} \geq \frac{r\left|b_{21}\right|}{2\left|b_{23}\right|}=\frac{c_{3}\left|b_{31}\right|}{2} \geq 3\left|b_{31}\right| .
$$

It follows that $b_{13}^{\prime}$ (resp., $b_{31}^{\prime}$ ) has the opposite sign to $b_{13}$ (resp., $b_{31}$ ). Thus, $B^{\prime}$ is cyclical, and it only remains to show that $B^{\prime}$ is 2-biased. To this effect, we note that $\left|b_{31}^{\prime}\right|=\left|b_{32} b_{21}\right|-\left|b_{31}\right|$, and so

$$
r^{\prime}=\left|b_{12}^{\prime} b_{23}^{\prime} b_{31}^{\prime}\right|=\left|b_{12}\right|\left|b_{23}\right|\left(\left|b_{32} b_{21}\right|-\left|b_{31}\right|\right)=c_{1} c_{3}-r \geq(r / 2) \cdot 6-r=2 r ;
$$

therefore, both $c_{1}^{\prime}=c_{1}$ and $c_{3}^{\prime}=c_{3}$ do not exceed $r^{\prime} / 2$. As for $c_{2}^{\prime}$, we have $\left|b_{13}^{\prime}\right|=\left|b_{12} b_{23}\right|-\left|b_{13}\right|$, and so

$$
c_{2}^{\prime}=\left|b_{13}^{\prime} b_{31}^{\prime}\right|=\left(\left|b_{12} b_{23}\right|-\left|b_{13}\right|\right)\left(\left|b_{32} b_{21}\right|-\left|b_{31}\right|\right)=r^{\prime}\left(1-\frac{c_{2}}{r}\right) ;
$$

since $c_{2} / r \leq 1 / 2$, we conclude that $r^{\prime}>r^{\prime}\left(1-\frac{c_{2}}{r}\right)=c_{2}^{\prime} \geq r^{\prime} / 2$. This completes the proof that $B^{\prime}$ is 2 -biased. Lemma 4.8 and Proposition 4.7 are proved. 


\section{EXChANGE RELATIONS: The COEFFICIENTS}

In this section we fix a family of matrices $B(t)$ satisfying the conditions in Proposition 4.3, and discuss possible choices of coefficients $p_{j}(t)$ that can appear in the corresponding exchange pattern. We start with the following simple characterization.

Proposition 5.1. Assume that matrices $(B(t))_{t \in \mathbb{T}_{n}}$ satisfy conditions (1)-(2) in Proposition 4.3. A family of elements $p_{j}(t)$ of a coefficient group $\mathbb{P}$ gives rise, via (4.2), to an exchange pattern if and only if they satisfy the following relations, whenever $t_{1} \stackrel{i}{-} t_{2} \stackrel{j}{\longrightarrow} t_{3} \stackrel{i}{\longrightarrow} t_{4}$ :

$$
p_{i}\left(t_{1}\right) p_{i}\left(t_{3}\right) p_{j}\left(t_{3}\right)^{\max \left(b_{j i}\left(t_{3}\right), 0\right)}=p_{i}\left(t_{2}\right) p_{i}\left(t_{4}\right) p_{j}\left(t_{2}\right)^{\max \left(b_{j i}\left(t_{2}\right), 0\right)} .
$$

Note that $b_{j i}\left(t_{2}\right)=-b_{j i}\left(t_{3}\right)$ by (4.3) $)$, so at most one of the $p_{j}\left(t_{2}\right)$ and $p_{j}\left(t_{3}\right)$ actually enters (5.1).

Proof. The only axiom of an exchange pattern that involves the coefficients is (2.7), and the relation (5.1) is precisely what it prescribes.

First of all, let us mention the trivial solution of (5.1) when all the coefficients $p_{i}(t)$ are equal to 1.

Moving in the opposite direction, we introduce the universal coefficient group (with respect to a fixed family $(B(t))$ ) as the abelian group $\mathbb{P}$ generated by the elements $p_{i}(t)$, for all $i \in I$ and $t \in \mathbb{T}_{n}$, which has (5.1) as the system of defining relations. The torsion-freeness of this group is guaranteed by the following proposition whose straightforward proof is omitted.

Proposition 5.2. The universal coefficient group $\mathbb{P}$ is a free abelian group. More specifically, let $t_{0} \in \mathbb{T}_{n}$, and let $S$ be a collection of pairs $(i, t)$ that contains both $\left(i, t_{0}\right)$ and $(i, t)$ for any edge $t_{0} \stackrel{i}{-} t$, and precisely one of the pairs $(i, t)$ and $\left(i, t^{\prime}\right)$

for each edge $t \stackrel{i}{-} t^{\prime}$ with $t$ and $t^{\prime}$ different from $t_{0}$. Then $\left\{p_{i}(t):(i, t) \in S\right\}$ is a set of free generators for $\mathbb{P}$.

We see that, in contrast to (4.3), relations (5.1) leave infinitely many degrees of freedom in determining the coefficients $p_{i}(t)$ (cf. Remark 2.2).

The rest of this section is devoted to important classes of exchange patterns within which all the coefficients are completely determined by specifying $2 n$ of them corresponding to the edges emanating from a given vertex.

Suppose that, in addition to the multiplicative group structure, the coefficient group $\mathbb{P}$ is supplied with a binary operation $\oplus$ that we call auxiliary addition. Furthermore, suppose that this operation is commutative, associative, and distributive with respect to multiplication; thus $(\mathbb{P}, \oplus, \cdot)$ is a semifield. (By the way, under these assumptions $\mathbb{P}$ is automatically torsion-free as a multiplicative group: indeed, if $p^{m}=1$ for some $p \in \mathbb{P}$ and $m \geq 2$, then

$$
\left.p=\frac{p^{m} \oplus p^{m-1} \oplus \cdots \oplus p}{p^{m-1} \oplus p^{m-2} \oplus \cdots \oplus 1}=\frac{p^{m-1} \oplus p^{m-2} \oplus \cdots \oplus 1}{p^{m-1} \oplus p^{m-2} \oplus \cdots \oplus 1}=1 .\right)
$$

Definition 5.3. An exchange pattern and the corresponding cluster algebra are called normalized if $\mathbb{P}$ is a semifield and $p_{j}(t) \oplus p_{j}\left(t^{\prime}\right)=1$ for any edge $t \stackrel{j}{j} t^{\prime}$. 
Proposition 5.4. Fix a vertex $t_{0} \in \mathbb{T}_{n}$ and $2 n$ elements $q_{j}$ and $r_{j}(j \in I)$ of a semifield $\mathbb{P}$ such that $q_{j} \oplus r_{j}=1$ for all $j$. Then every family of matrices $B(t)$ satisfying the conditions in Proposition 4.3 gives rise to a unique normalized exchange pattern such that, for every edge $t_{0} \stackrel{j}{-} t$, we have $p_{j}\left(t_{0}\right)=q_{j}$ and $p_{j}(t)=r_{j}$. Thus a normalized exchange pattern is completely determined by the $2 n$ monomials $M_{j}\left(t_{0}\right)$ and $M_{j}(t)$, for all edges $t_{0} \stackrel{j}{\longrightarrow} t$.

Proof. In a normalized exchange pattern, the coefficients $p_{j}(t)$ and $p_{j}\left(t^{\prime}\right)$ corresponding to an edge $t \stackrel{j}{j} t^{\prime}$ are determined by their ratio

$$
u_{j}(t)=\frac{p_{j}(t)}{p_{j}\left(t^{\prime}\right)}
$$

via

$$
p_{j}(t)=\frac{u_{j}(t)}{1 \oplus u_{j}(t)}, \quad p_{j}\left(t^{\prime}\right)=\frac{1}{1 \oplus u_{j}(t)} .
$$

Clearly, we have

$$
u_{j}(t) u_{j}\left(t^{\prime}\right)=1
$$

for any edge $t \stackrel{j}{-} t^{\prime}$. We can also rewrite the relation (5.1) as follows:

$$
u_{i}\left(t^{\prime}\right)=u_{i}(t) u_{j}(t)^{\max \left(b_{j i}(t), 0\right)}\left(1 \oplus u_{j}(t)\right)^{-b_{j i}(t)}
$$

for any edge $t \stackrel{j}{j} t^{\prime}$ and any $i \neq j$. This form of the relations for the normalized coefficients makes our proposition obvious.

Remark 5.5. It is natural to ask whether the normalization condition imposes additional multiplicative relations among the coefficients $p_{i}(t)$ that are not consequences of (5.1). In other words: can a normalized system of coefficients generate the universal coefficient group? In the next section, we present a complete answer to this question in the rank 2 case (see Remark 6.5 below).

One example of a semifield is the multiplicative group $\mathbb{R}_{>0}$ of positive real numbers, $\oplus$ being ordinary addition. However the following example is more important for our purposes.

Example 5.6. Let $\mathbb{P}$ be a free abelian group, written multiplicatively, with a finite set of generators $p_{i}\left(i \in I^{\prime}\right)$, and with auxiliary addition $\oplus$ defined by

$$
\prod_{i} p_{i}^{a_{i}} \oplus \prod_{i} p_{i}^{b_{i}}=\prod_{i} p_{i}^{\min \left(a_{i}, b_{i}\right)} .
$$

Then $\mathbb{P}$ is a semifield; specifically, it is a product of $\left|I^{\prime}\right|$ copies of the tropical semifield (see, e.g., [1]). We denote this semifield by $\operatorname{Trop}\left(p_{i}: i \in I^{\prime}\right)$. Note that if all exponents $a_{i}$ and $b_{i}$ in (5.6) are nonnegative, then the monomial on the righthand side is the gcd of the two monomials on the left.

Definition 5.7. We say that an exchange pattern is of geometric type if it is normalized, has the coefficient semifield $\mathbb{P}=\operatorname{Trop}\left(p_{i}: i \in I^{\prime}\right)$, and each coefficient $p_{j}(t)$ is a monomial in the generators $p_{i}$ with all exponents nonnegative. 
For an exchange pattern of geometric type, the normalization condition simply means that, for every edge $t \stackrel{j}{\prime} t^{\prime}$, the two monomials $p_{j}(t)$ and $p_{j}\left(t^{\prime}\right)$ in the generators $p_{i}$ are coprime, i.e., have no variable in common.

In all our examples of cluster algebras of geometric origin, including those discussed in the introduction, the exchange patterns turn out to be of geometric type. These patterns have the following useful equivalent description.

Proposition 5.8. Let $\mathbb{P}=\operatorname{Trop}\left(p_{i}: i \in I^{\prime}\right)$. A family of coefficients $p_{j}(t) \in \mathbb{P}$ gives rise to an exchange pattern of geometric type if and only if they are given by

$$
p_{j}(t)=\prod_{i \in I^{\prime}} p_{i}^{\max \left(c_{i j}(t), 0\right)}
$$

for some family of integers $\left(c_{i j}(t): t \in \mathbb{T}_{n}, i \in I^{\prime}, j \in I\right)$ ) satisfying the following property: for every edge $t \stackrel{k}{-} t^{\prime}$ in $\mathbb{T}_{n}$, the matrices $C(t)=\left(c_{i j}(t)\right)=\left(c_{i j}\right)$ and $C\left(t^{\prime}\right)=\left(c_{i j}\left(t^{\prime}\right)\right)=\left(c_{i j}^{\prime}\right)$ are related by

$$
c_{i j}^{\prime}= \begin{cases}-c_{i j} & \text { if } j=k ; \\ c_{i j}+\frac{\left|c_{i k}\right| b_{k j}(t)+c_{i k}\left|b_{k j}(t)\right|}{2} & \text { otherwise. }\end{cases}
$$

Proof. As in the proof of Proposition 5.4, for every edge $t \frac{j}{1} t^{\prime}$ we consider the ratio $u_{j}(t)=p_{j}(t) / p_{j}\left(t^{\prime}\right)$. We introduce the matrices $C(t)$ by setting

$$
u_{j}(t)=\prod_{i} p_{i}^{c_{i j}(t)} .
$$

The expression (5.7) then becomes a specialization of the first equality in (5.3), with auxiliary addition given by (5.6). To derive (5.8) from (5.5), first replace $i$ by $j$ and $j$ by $k$, respectively, then specialize, then pick up the exponent of $p_{i}$.

Comparing (5.8) with (4.3), we see that it is natural to combine a pair of matrices $(B(t), C(t))$ into one rectangular integer matrix $\tilde{B}(t)=\left(b_{i j}\right)_{i \in I \amalg I^{\prime}, j \in I}$ by setting $b_{i j}=c_{i j}$ for $i \in I^{\prime}$ and $j \in I$. Then the matrices $\tilde{B}(t)$ for $t \in \mathbb{T}_{n}$ are related to each other by the same matrix mutation rule (4.3), now applied to any $i \in I \coprod I^{\prime}$ and $j \in I$. We refer to $B(t)$ as the principal part of $\tilde{B}(t)$. Combining Propositions 5.8 and 4.5 we obtain the following corollary.

Corollary 5.9. Let $\tilde{B}$ be an integer matrix with skew-symmetrizable principal part. There is a unique exchange pattern $\mathcal{M}=\mathcal{M}(\tilde{B})$ of geometric type such that $\tilde{B}\left(t_{0}\right)=$ $\tilde{B}$ at a given vertex $t_{0} \in \mathbb{T}_{n}$.

In the geometric type case, there is a distinguished choice of ground ring for the corresponding cluster algebra: take $\mathbb{A}$ to be the polynomial ring $\mathbb{Z}\left[p_{i}: i \in I^{\prime}\right]$. In the situation of Corollary 5.9 , we will denote the corresponding cluster algebra $\mathcal{A}_{\mathbb{A}}(\mathcal{M})$ simply by $\mathcal{A}(\tilde{B})$. In the notation of Section $2, \mathcal{A}(\tilde{B})$ is the subring of the ambient field $\mathcal{F}$ generated by cluster variables $x_{j}(t)$ for all $j \in I$ and $t \in \mathbb{T}_{n}$ together with the generators $p_{i}\left(i \in I^{\prime}\right)$ of $\mathbb{P}$.

The set $I^{\prime}$ is allowed to be empty: this simply means that all the coefficients $p_{j}(t)$ in the corresponding exchange pattern are equal to 1 . In this case, we have $\tilde{B}(t)=B(t)$ for all $t$. 
We note that the class of exchange patterns of geometric type (and the corresponding cluster algebras $\mathcal{A}(\tilde{B}))$ is stable under the operations of restriction and direct product introduced in Section 2 The restriction from $I$ to a subset $J$ amounts to removing from $\tilde{B}$ the columns labeled by $I-J$; the direct product operation replaces two matrices $\tilde{B}_{1}$ and $\tilde{B}_{2}$ by the matrix $\left[\begin{array}{cc}\tilde{B}_{1} & 0 \\ 0 & \tilde{B}_{2}\end{array}\right]$.

\section{THE RANK 2 CASE}

In this section, we illustrate the above results and constructions by treating in detail the special case $n=2$. We label vertices and edges of the tree $\mathbb{T}_{2}$ as in (2.10). For $m \in \mathbb{Z}$, it will be convenient to denote by $\langle m\rangle$ the element of $\{1,2\}$ congruent to $m$ modulo 2 . Thus, $\mathbb{T}_{2}$ consists of vertices $t_{m}$ and edges $t_{m} \frac{\langle m\rangle}{t_{m+1}}$ for all $m \in \mathbb{Z}$. We use the notation of Example 2.5, so the clusters are of the form $\mathbf{x}\left(t_{m}\right)=\left\{y_{m}, y_{m+1}\right\}$, and the exchange relations are given by (2.11), with coefficients $q_{m}$ and $r_{m}$ satisfying (2.12). More specifically, we have

$$
x_{\langle m\rangle}\left(t_{m}\right)=y_{m}, x_{\langle m+1\rangle}\left(t_{m}\right)=y_{m+1}, p_{\langle m\rangle}\left(t_{m}\right)=q_{m+1}, p_{\langle m+1\rangle}\left(t_{m}\right)=r_{m}
$$

for $m \in \mathbb{Z}$. Choose $\mathbf{x}\left(t_{1}\right)=\left\{y_{1}, y_{2}\right\}$ as the initial cluster. According to Theorem 3.1 each cluster variable $y_{m}$ can be expressed as a Laurent polynomial in $y_{1}$ and $y_{2}$ with coefficients in $\mathbb{Z} \mathbb{P}$. Let us write this Laurent polynomial as

$$
y_{m}=\frac{N_{m}\left(y_{1}, y_{2}\right)}{y_{1}^{d_{1}(m)} y_{2}^{d_{2}(m)}}
$$

where $N_{m}\left(y_{1}, y_{2}\right) \in \mathbb{Z} \mathbb{P}\left[y_{1}, y_{2}\right]$ is a polynomial not divisible by $y_{1}$ or $y_{2}$. We will investigate in detail the denominators of these Laurent polynomials.

Recall that for $n=2$ the matrices $B(t)$ are given by (4.5). Thus, all these matrices have the same associated Cartan matrix

$$
A=A(B(t))=\left[\begin{array}{cc}
2 & -b \\
-c & 2
\end{array}\right]
$$

(see (4.6)). We will show that the denominators in (6.2) have a nice interpretation in terms of the root system associated to $A$. Let us recall some basic facts about this root system (cf., e.g., [10]).

Let $Q \cong \mathbb{Z}^{2}$ be a lattice of rank 2 with a fixed basis $\left\{\alpha_{1}, \alpha_{2}\right\}$ of simple roots. The Weyl group $W=W(A)$ is a group of linear transformations of $Q$ generated by two simple reflections $s_{1}$ and $s_{2}$ whose action in the basis of simple roots is given by

$$
s_{1}=\left[\begin{array}{cc}
-1 & b \\
0 & 1
\end{array}\right], \quad s_{2}=\left[\begin{array}{cc}
1 & 0 \\
c & -1
\end{array}\right] .
$$

Since both $s_{1}$ and $s_{2}$ are involutions, each element of $W$ is one of the following:

$$
w_{1}(m)=s_{1} s_{2} s_{1} \cdots s_{\langle m\rangle}, w_{2}(m)=s_{2} s_{1} s_{2} \cdots s_{\langle m+1\rangle} ;
$$

here both products are of length $m \geq 0$. It is well known that $W$ is finite if and only if $b c \leq 3$; we shall refer to this as the finite case. The Coxeter number $h$ of $W$ is the order of $s_{1} s_{2}$ in $W$; it is given by Table 1 In the finite case, $W$ is the dihedral group of order $2 h$, and its elements can be listed as follows: $w_{1}(0)=w_{2}(0)=e$ 
(the identity element), $w_{1}(h)=w_{2}(h)=w_{0}$ (the longest element), and $2 h-2$ distinct elements $w_{1}(m), w_{2}(m)$ for $0<m<h$. In the infinite case, all elements $w_{1}(m)$ and $w_{2}(m)$ for $m>0$ are distinct.

TABle 1 . The Coxeter number

\begin{tabular}{|c|c|c|c|c|c|}
\hline$b c$ & 0 & 1 & 2 & 3 & $\geq 4$ \\
\hline$h$ & 2 & 3 & 4 & 6 & $\infty$ \\
\hline
\end{tabular}

A vector $\alpha \in Q$ is a real root for $A$ if it is $W$-conjugate to a simple root. Let $\Phi$ denote the set of real roots for $A$. It is known that $\Phi=\Phi_{+} \cup\left(-\Phi_{+}\right)$, where

$$
\Phi_{+}=\left\{\alpha=d_{1} \alpha_{1}+d_{2} \alpha_{2} \in \Phi: d_{1}, d_{2} \geq 0\right\}
$$

is the set of positive real roots. In the finite case, $\Phi_{+}$has cardinality $h$, and we have

$$
\Phi_{+}=\left\{w_{1}(m) \alpha_{\langle m+1\rangle}: 0 \leq m<h\right\} .
$$

In the infinite case, we have

$$
\Phi_{+}=\left\{w_{1}(m) \alpha_{\langle m+1\rangle}, w_{2}(m) \alpha_{\langle m+2\rangle}: m \geq 0\right\},
$$

with all the roots $w_{1}(m) \alpha_{\langle m+1\rangle}$ and $w_{2}(m) \alpha_{\langle m+2\rangle}$ distinct.

We will represent the denominators in (6.2) as vectors in the root lattice $Q$ by setting

$$
\delta(m)=d_{1}(m) \alpha_{1}+d_{2}(m) \alpha_{2}
$$

for all $m \in \mathbb{Z}$. In particular, we have $\delta(1)=-\alpha_{1}$ and $\delta(2)=-\alpha_{2}$.

Theorem 6.1. In the rank 2 case (finite or infinite alike), cluster variables are uniquely up to a multiple from $\mathbb{P}$ determined by their denominators in the Laurent expansions with respect to a given cluster. The set of these denominators is naturally identified with $\left\{-\alpha_{1},-\alpha_{2}\right\} \cup \Phi_{+}$. More precisely:

(i) In the infinite case, we have

$$
\delta(m+3)=w_{1}(m) \alpha_{\langle m+1\rangle}, \quad \delta(-m)=w_{2}(m) \alpha_{\langle m+2\rangle} \quad(m \geq 0) .
$$

In particular, all $y_{m}$ for $m \in \mathbb{Z}$ have different denominators $y_{1}^{d_{1}(m)} y_{2}^{d_{2}(m)}$.

(ii) In the finite case, we have

$$
\delta(m+3)=w_{1}(m) \alpha_{\langle m+1\rangle} \quad(h>m \geq 0)
$$

and $\delta(m+h+2)=\delta(m)$ for all $m \in \mathbb{Z}$, so the denominators $y_{1}^{d_{1}(m)} y_{2}^{d_{2}(m)}$ are periodic with the period $h+2$. Moreover, $y_{m+h+2} / y_{m} \in \mathbb{P}$ for $m \in \mathbb{Z}$.

Proof. Before giving a unified proof of Theorem 6.1, we notice that part (ii) can be proved by a direct calculation. We will express all coefficients in terms of $r_{1}$, $r_{2}$, and $q_{m}$ for $m \in \mathbb{Z}$ (cf. Proposition 5.2). With the help of Maple we find that in each finite case, the elements $y_{m}$ for $3 \leq m \leq h+4$ are the following Laurent polynomials in $y_{1}$ and $y_{2}$.

Type $A_{1} \times A_{1}: b=c=0$.

$$
y_{3}=\frac{q_{2}+r_{2}}{y_{1}}, \quad y_{4}=\frac{q_{3}\left(q_{1}+r_{1}\right)}{r_{1} y_{2}}, \quad y_{5}=\frac{q_{4} y_{1}}{r_{2}}, \quad y_{6}=\frac{r_{1} q_{5} y_{2}}{q_{1} q_{3}} .
$$


Type $A_{2}: b=c=1$.

$$
\begin{gathered}
y_{3}=\frac{q_{2}+r_{2}}{y_{1}}, \quad y_{4}=\frac{q_{3}\left(r_{2}\left(q_{1} y_{1}+r_{1}\right)+r_{1} q_{2} y_{2}\right)}{r_{1} y_{1} y_{2}}, \\
y_{5}=\frac{q_{3} q_{4}\left(q_{1} y_{1}+r_{1}\right)}{r_{1} y_{2}}, \quad y_{6}=\frac{q_{4} q_{5} y_{1}}{r_{2}}, \quad y_{7}=\frac{r_{1} q_{5} q_{6} y_{2}}{r_{2} q_{1} q_{3}} .
\end{gathered}
$$

Type $B_{2}: b=1, c=2$.

$$
\begin{gathered}
y_{3}=\frac{q_{2} y_{2}^{2}+r_{2}}{y_{1}}, \quad y_{4}=\frac{q_{3}\left(r_{2}\left(q_{1} y_{1}+r_{1}\right)+r_{1} q_{2} y_{2}^{2}\right)}{r_{1} y_{1} y_{2}} \\
y_{5}=\frac{q_{3}^{2} q_{4}\left(r_{2}\left(q_{1} y_{1}+r_{1}\right)^{2}+r_{1}^{2} q_{2} y_{2}^{2}\right)}{r_{1}^{2} y_{1} y_{2}^{2}}, \quad y_{6}=\frac{q_{3} q_{4} q_{5}\left(q_{1} y_{1}+r_{1}\right)}{r_{1} y_{2}}, \\
y_{7}=\frac{q_{4} q_{5}^{2} q_{6} y_{1}}{r_{2}}, \quad y_{8}=\frac{r_{1} q_{5} q_{6} q_{7} y_{2}}{r_{2} q_{1} q_{3}}
\end{gathered}
$$

Type $C_{2}: b=2, c=1$.

$$
\begin{gathered}
y_{3}=\frac{q_{2} y_{2}+r_{2}}{y_{1}}, \quad y_{4}=\frac{q_{3}\left(r_{2}^{2} q_{1} y_{1}^{2}+r_{1}\left(q_{2} y_{2}+r_{2}\right)^{2}\right)}{r_{1} y_{1}^{2} y_{2}}, \\
y_{5}=\frac{q_{3} q_{4}\left(r_{2} q_{1} y_{1}^{2}+r_{1}\left(q_{2} y_{2}+r_{2}\right)\right)}{r_{1} y_{1} y_{2}}, \quad y_{6}=\frac{q_{3} q_{4}^{2} q_{5}\left(q_{1} y_{1}^{2}+r_{1}\right)}{r_{1} y_{2}}, \\
y_{7}=\frac{q_{4} q_{5} q_{6} y_{1}}{r_{2}}, \quad y_{8}=\frac{r_{1} q_{5} q_{6}^{2} q_{7} y_{2}}{r_{2}^{2} q_{1} q_{3}} .
\end{gathered}
$$

Type $G_{2}: b=1, c=3$.

$$
\begin{gathered}
y_{3}=\frac{q_{2} y_{2}^{3}+r_{2}}{y_{1}}, \quad y_{4}=\frac{q_{3}\left(r_{2}\left(q_{1} y_{1}+r_{1}\right)+r_{1} q_{2} y_{2}^{3}\right)}{r_{1} y_{1} y_{2}} \\
y_{5}=\frac{q_{3}^{3} q_{4}\left(r_{2}^{2}\left(q_{1} y_{1}+r_{1}\right)^{3}+r_{1}^{2} q_{2} y_{2}^{3}\left(r_{1} q_{2} y_{2}^{3}+3 r_{2} q_{1} y_{1}+2 r_{1} r_{2}\right)\right)}{r_{1}^{3} y_{1}^{2} y_{2}^{3}} \\
y_{6}=\frac{q_{3}^{2} q_{4} q_{5}\left(r_{2}\left(q_{1} y_{1}+r_{1}\right)^{2}+r_{1}^{2} q_{2} y_{2}^{3}\right)}{r_{1}^{2} y_{1} y_{2}^{2}}, \quad y_{7}=\frac{q_{3}^{3} q_{4}^{2} q_{5}^{3} q_{6}\left(r_{2}\left(q_{1} y_{1}+r_{1}\right)^{3}+r_{1}^{3} q_{2} y_{2}^{3}\right)}{r_{1}^{3} y_{1} y_{2}^{3}}, \\
y_{8}=\frac{q_{3} q_{4} q_{5}^{2} q_{6} q_{7}\left(q_{1} y_{1}+r_{1}\right)}{r_{1} y_{2}}, \quad y_{9}=\frac{q_{4} q_{5}^{3} q_{6}^{2} q_{7}^{3} q_{8} y_{1}}{r_{2}}, \quad y_{10}=\frac{r_{1} q_{5} q_{6} q_{7}^{2} q_{8} q_{9} y_{2}}{r_{2} q_{1} q_{3}} .
\end{gathered}
$$

Type $G_{2}^{\vee}: b=3, c=1$.

$$
\begin{gathered}
y_{3}=\frac{q_{2} y_{2}+r_{2}}{y_{1}}, \quad y_{4}=\frac{q_{3}\left(r_{1}\left(q_{2} y_{2}+r_{2}\right)^{3}+r_{2}^{3} q_{1} y_{1}^{3}\right)}{r_{1} y_{1}^{3} y_{2}}, \\
y_{5}=\frac{q_{3} q_{4}\left(r_{1}\left(q_{2} y_{2}+r_{2}\right)^{2}+r_{2}^{2} q_{1} y_{1}^{3}\right)}{r_{1} y_{1}^{2} y_{2}}, \\
y_{6}=\frac{q_{3}^{2} q_{4}^{3} q_{5}\left(r_{1}^{2}\left(q_{2} y_{2}+r_{2}\right)^{3}+r_{2}^{2} q_{1} y_{1}^{3}\left(r_{2} q_{1} y_{1}^{3}+3 r_{1} q_{2} y_{2}+2 r_{1} r_{2}\right)\right)}{r_{1}^{2} y_{1}^{3} y_{2}^{2}},
\end{gathered}
$$




$$
\begin{gathered}
y_{7}=\frac{q_{3} q_{4}^{2} q_{5} q_{6}\left(r_{2}\left(q_{1} y_{1}^{3}+r_{1}\right)+r_{1} q_{2} y_{2}\right)}{r_{1} y_{1} y_{2}}, \quad y_{8}=\frac{q_{3} q_{4}^{3} q_{5}^{2} q_{6}^{3} q_{7}\left(q_{1} y_{1}^{3}+r_{1}\right)}{r_{1} y_{2}} \\
y_{9}=\frac{q_{4} q_{5} q_{6}^{2} q_{7} q_{8} y_{1}}{r_{2}}, \quad y_{10}=\frac{r_{1} q_{5} q_{6}^{3} q_{7}^{2} q_{8}^{3} q_{9} y_{2}}{r_{2}^{3} q_{1} q_{3}}
\end{gathered}
$$

By inspection, the exponents of the $y$ variables in the denominators above agree with (6.6) . Furthermore, we see that $y_{m+h+2} / y_{m} \in \mathbb{P}$ for $m=1,2$, and therefore for any $m$.

Now let us give a unified proof of both parts of Theorem 6.1 For $\delta=d_{1} \alpha_{1}+$ $d_{2} \alpha_{2} \in Q$, let us denote

$$
[\delta]_{+}=\max \left(d_{1}, 0\right) \alpha_{1}+\max \left(d_{2}, 0\right) \alpha_{2} .
$$

It then follows from the relations (2.11) that

$$
\delta(m+1)+\delta(m-1)= \begin{cases}b[\delta(m)]_{+} & \text {if } m \text { is odd } \\ c[\delta(m)]_{+} & \text {if } m \text { is even }\end{cases}
$$

Starting with $\delta(1)=-\alpha_{1}$ and $\delta(2)=-\alpha_{2}$, we use (6.7) to see that

$$
\delta(3)=\alpha_{1}, \quad \delta(4)=b \alpha_{1}+\alpha_{2}=s_{1} \alpha_{2}
$$

and also

$$
\delta(0)=\alpha_{2}, \quad \delta(-1)=\alpha_{1}+c \alpha_{2}=s_{2} \alpha_{1} .
$$

This proves (6.5) and (6.6) for $m=0$ and $m=1$.

Proceeding by induction on $m$, let us now assume that $m \geq 2$, and that both vectors $\delta(m+1)$ and $\delta(m+2)$ are positive roots given by (6.6); in the finite case, we also assume that $m \leq h$. If $m$ is odd, it follows from (6.7) that

$$
\begin{aligned}
\delta(m+3) & =b \delta(m+2)-\delta(m+1)=b w_{1}(m-1) \alpha_{1}-w_{1}(m-2) \alpha_{2} \\
& =w_{1}(m-1)\left(s_{1} \alpha_{2}-\alpha_{2}\right)-w_{1}(m-2) \alpha_{2} \\
& =w_{1}(m) \alpha_{2}-w_{1}(m-2)\left(s_{2} \alpha_{2}+\alpha_{2}\right)=w_{1}(m) \alpha_{2} .
\end{aligned}
$$

Thus, $\delta(m+3)$ is also given by ([6.6]). The same argument works with $m$ even, and, in the infinite case, with $m$ negative. This completes the proof of $(6.5)$ and $(6.6)$.

We conclude by a unified proof of the periodicity $\delta(m+h+2)=\delta(m)$ in the finite case. Without loss of generality, we assume that $m=1$. By the above inductive argument, we know that $\delta(h+3)$ is given by $\underline{6.6}$. Thus, we have $\delta(h+3)=$ $w_{1}(h) \alpha_{\langle h+1\rangle}=w_{0} \alpha_{\langle h+1\rangle}$. It is known-and easy to check - that $w_{0}=\left[\begin{array}{cc}0 & -1 \\ -1 & 0\end{array}\right]$ if $b=c=1$ (the only case when $h$ is odd), and $w_{0}=\left[\begin{array}{cc}-1 & 0 \\ 0 & -1\end{array}\right]$ in the other three finite cases. Hence $w_{0} \alpha_{\langle h+1\rangle}=-\alpha_{1}$ in all cases, and we are done.

In the rest of the section, we discuss the normalized case (see Definition 5.3). In this context, the periodicity property in Theorem 6.1 (ii) can be sharpened considerably.

Proposition 6.2. In the normalized finite case of a cluster algebra of rank 2 , we have $q_{m+h+2}=q_{m}, r_{m+h+2}=r_{m}$, and $y_{m+h+2}=y_{m}$ for all $m \in \mathbb{Z}$. 
Proof. We set $u_{m}=q_{m} / r_{m}$ for $m \in \mathbb{Z}$. Then $q_{m}$ and $r_{m}$ are recovered from $u_{m}$ by

$$
q_{m}=\frac{u_{m}}{1 \oplus u_{m}}, \quad r_{m}=\frac{1}{1 \oplus u_{m}}
$$

(cf. (5.3)). To establish the periodicity of $q_{m}$ and $r_{m}$, it suffices to show that $u_{m+h+2}=u_{m}$. Furthermore, it is enough to check that $u_{h+3}=u_{1}$ and $u_{h+4}=u_{2}$ in each of the following four cases: $(b, c)=(0,0)$ (type $\left.A_{1} \times A_{1}\right),(1,1)$ (type $A_{2}$ ), $(1,2)$ (type $\left.B_{2}\right)$, and $(1,3)$ (type $\left.G_{2}\right)$. This is done by direct calculation using the relation

$$
u_{m+1} u_{m-1}= \begin{cases}\left(1 \oplus u_{m}\right)^{c} & \text { if } m \text { is odd } \\ \left(1 \oplus u_{m}\right)^{b} & \text { if } m \text { is even, }\end{cases}
$$

which, in view of (6.1), is a consequence of (5.4) and (5.5). Below are the intermediate steps of this calculation, which in each case expresses $u_{m}$ for $3 \leq m \leq h+2$ as a rational function in $u_{1}$ and $u_{2}$, and then confirms $u_{h+3}=u_{1}$ and $u_{h+4}=u_{2}$.

Type $A_{1} \times A_{1}: b=c=0, h=2$.

$$
u_{3}=\frac{1}{u_{1}}, \quad u_{4}=\frac{1}{u_{2}} .
$$

Type $A_{2}: b=c=1, h=3$.

$$
u_{3}=\frac{1 \oplus u_{2}}{u_{1}}, \quad u_{4}=\frac{1 \oplus u_{1} \oplus u_{2}}{u_{1} u_{2}}, \quad u_{5}=\frac{1 \oplus u_{1}}{u_{2}} .
$$

Type $B_{2}: b=1, c=2, h=4$.

$$
u_{3}=\frac{1 \oplus u_{2}}{u_{1}}, \quad u_{4}=\frac{\left(1 \oplus u_{1} \oplus u_{2}\right)^{2}}{u_{1}^{2} u_{2}}, \quad u_{5}=\frac{\left(1 \oplus u_{1}\right)^{2} \oplus u_{2}}{u_{1} u_{2}}, \quad u_{6}=\frac{\left(1 \oplus u_{1}\right)^{2}}{u_{2}} .
$$

Type $G_{2}: b=1, c=3, h=6$.

$$
\begin{gathered}
u_{3}=\frac{1 \oplus u_{2}}{u_{1}}, \quad u_{4}=\frac{\left(1 \oplus u_{1} \oplus u_{2}\right)^{3}}{u_{1}^{3} u_{2}}, \quad u_{5}=\frac{\left(1 \oplus u_{1}\right)^{3} \oplus u_{2}\left(3 u_{1} \oplus u_{2} \oplus 2\right)}{u_{1}^{2} u_{2}}, \\
u_{6}=\frac{\left(\left(1 \oplus u_{1}\right)^{2} \oplus u_{2}\right)^{3}}{u_{1}^{3} u_{2}^{2}}, \quad u_{7}=\frac{\left(1 \oplus u_{1}\right)^{3} \oplus u_{2}}{u_{1} u_{2}}, \quad u_{8}=\frac{\left(1 \oplus u_{1}\right)^{3}}{u_{2}} .
\end{gathered}
$$

To complete the proof of Proposition 6.2, it remains to show that $y_{h+3}=y_{1}$ and $y_{h+4}=y_{2}$ in each of the four cases. By Theorem 6.1 (ii), both ratios $y_{h+3} / y_{1}$ and $y_{h+4} / y_{2}$ belong to $\mathbb{P}$, and we only need to show that these two elements of $\mathbb{P}$ are equal to 1 in the normalized case. The ratios in question were explicitly computed in the course of the proof of Theorem 6.1. We see that it all boils down to the following identities:

$$
\begin{aligned}
& \text { Type } A_{1} \times A_{1}: r_{1}=q_{3} . \\
& \text { Type } A_{2}: r_{1}=q_{3} q_{4} . \\
& \text { Type } B_{2}: r_{1}=q_{3} q_{4} q_{5}, r_{2}=q_{4} q_{5}^{2} q_{6} . \\
& \text { Type } G_{2}: r_{1}=q_{3} q_{4} q_{5}^{2} q_{6} q_{7}, r_{2}=q_{4} q_{5}^{3} q_{6}^{2} q_{7}^{3} q_{8} .
\end{aligned}
$$

All these identities can be proved by a direct calculation (preferably, with a computer): first express both sides in terms of the $u_{m}$ using (6.8), and then replace each $u_{m}$ by the above expression in terms of $u_{1}$ and $u_{2}$. 
Remark 6.3. Periodicity of the recurrence (6.9) is a very special case of the periodicity phenomenon for $Y$-system recurrences in the theory of the thermodynamic Bethe ansatz [19]. We plan to address the case of arbitrary rank in a forthcoming paper.

We have shown that in a finite normalized case, any coefficient $r_{m}$ can be written as a monomial in $q_{m+2}, \ldots, q_{m+h}$. There is a nice uniform way to write down these monomials using the dual root system of the system $\Phi$ considered above. Recall that the dual root system $\Phi^{\vee}$ is associated with the transposed Cartan matrix. The simple roots of $\Phi^{\vee}$ are called simple coroots and denoted $\alpha_{1}^{\vee}$ and $\alpha_{2}^{\vee}$. They generate the coroot lattice $Q^{\vee}$. The Weyl groups of $\Phi$ and $\Phi^{\vee}$ are naturally isomorphic to each other, and it is common to identify them. The same Weyl group $W$ acts on $Q^{\vee}$ so that the action of simple reflections $s_{1}$ and $s_{2}$ in the basis of simple coroots is given by the matrices

$$
s_{1}=\left[\begin{array}{cc}
-1 & c \\
0 & 1
\end{array}\right], \quad s_{2}=\left[\begin{array}{cc}
1 & 0 \\
b & -1
\end{array}\right]
$$

(cf. (6.4)). By inspection (cf. (6.10) 6.13) ), one obtains the following unified expression for $r_{m}$.

Proposition 6.4. For $i \in\{1,2\}$ and $m \geq 0$, let $c(i, m)$ denote the coefficient of $\alpha_{i}^{\vee}$ in the root $w_{i}(m) \alpha_{\langle i+m\rangle}^{\vee}$. Then, in every finite case, we have

$$
r_{k}=\prod_{m=0}^{h-2} q_{k+m+2}^{c(\langle k+1\rangle, m)}
$$

for all $k \in \mathbb{Z}$.

Remark 6.5. The relations (6.14) together with the periodicity relation $q_{m+h+2}=$ $q_{m}$ imply that in each finite normalized case the subgroup of the coefficient group $\mathbb{P}$ generated by all $q_{m}$ and $r_{m}$ is in fact generated by $q_{1}, \ldots, q_{h+2}$. Thus, this group is dramatically different from the universal coefficient group. One might ask if the normalization condition imposes some relations among $q_{1}, \ldots, q_{h+2}$. The answer to this question is negative: it is possible to take $\mathbb{P}=\operatorname{Trop}\left(q_{1}, \ldots, q_{h+2}\right)$ (cf. Example [5.6), so that multiplicatively, $\mathbb{P}$ is a free abelian group generated by $q_{1}, \ldots, q_{h+2}$. With the help of (6.14) and the periodicity relation $q_{m+h+2}=q_{m}$, we can view all $r_{m}$ and $q_{m}$ for $m \in \mathbb{Z}$ as elements of $\mathbb{P}$. By the definition of auxiliary addition (see (5.6) ), we have $r_{m} \oplus q_{m}=1$ for all $m$. Hence these coefficients give rise to a normalized exchange pattern (which is in fact of geometric type according to Definition 5.7).

In the infinite case, the situation is very different: there exists a normalized exchange pattern such that the coefficients $r_{m}$ and $q_{m}$ generate the universal coefficient group (see Proposition 5.2). To see this, take $\mathbb{P}$ to be the group of formal infinite products $\prod_{m \in \mathbb{Z}} q_{m}^{c_{m}}$, where the exponents $c_{m}$ can be arbitrary integers. Make $\mathbb{P}$ into a semifield by setting

$$
\prod_{m \in \mathbb{Z}} q_{m}^{c_{m}} \oplus \prod_{m \in \mathbb{Z}} q_{m}^{c_{m}^{\prime}}=\prod_{m \in \mathbb{Z}} q_{m}^{\min \left(c_{m}, c_{m}^{\prime}\right)}
$$

Taking (6.14) as an inspiration, let us define the elements $r_{k} \in \mathbb{P}$ for all $k \in \mathbb{Z}$ by

$$
r_{k}=\prod_{m \geq 0}\left(q_{k+m+2} q_{k-m-2}\right)^{c(\langle k+1\rangle, m)},
$$


with the same exponents as in (6.14). Also, view each $q_{k}$ as an element of $\mathbb{P}$ via $q_{k}=\prod_{m \in \mathbb{Z}} q_{m}^{\delta_{k m}}$. A direct check then shows that these coefficients give rise to a normalized exchange pattern (that is, satisfy $(2.12)$ ), and also that in $\mathbb{P}$ there are no relations among the elements $r_{1}, r_{2}$, and $q_{k}$, for $k \in \mathbb{Z}$.

\section{THE EXCHANGE GRAPH}

In this section, we restrict our attention to normalized exchange patterns $\mathcal{M}=$ $\left(M_{j}(t): j \in I, t \in \mathbb{T}_{n}\right)$. Let $\left(x_{j}(t): j \in I, t \in \mathbb{T}_{n}\right)$ be the family of cluster variables associated to $\mathcal{M}$. As in Section 2, we view them as elements of the ambient field $\mathcal{F}$.

A normalized exchange pattern gives rise to a natural equivalence relation on the set of vertices of $\mathbb{T}_{n}$. Informally speaking, two vertices are equivalent with respect to $\mathcal{M}$ if they have the same clusters and the same exchange relations, up to relabeling of cluster variables. Here is a precise definition.

Definition 7.1. We say that two vertices $t$ and $t^{\prime}$ in $\mathbb{T}_{n}$ are $\mathcal{M}$-equivalent if there exists a permutation $\sigma$ of the index set $I$ such that:

(1) $x_{i}\left(t^{\prime}\right)=x_{\sigma(i)}(t)$ for all $i \in I$;

(2) if $t \frac{\sigma(j)}{\underline{a}} t_{1}$ and $t^{\prime} \stackrel{j}{-} t_{1}^{\prime}$ for some $j \in I$, then $M_{j}\left(t^{\prime}\right)\left(\mathbf{x}\left(t^{\prime}\right)\right)=M_{\sigma(j)}(t)(\mathbf{x}(t))$ and $M_{j}\left(t_{1}^{\prime}\right)\left(\mathbf{x}\left(t^{\prime}\right)\right)=M_{\sigma(j)}\left(t_{1}\right)(\mathbf{x}(t))$.

Remark 7.2. We believe that condition (2) in Definition 7.1 is in fact a consequence of (1). Thus, once a cluster has been fixed as a set (forgetting the labels and the cluster's location in the tree $\mathbb{T}_{n}$ ), the exchange relations involving its elements are uniquely determined.

In view of Proposition 5.4, a normalized exchange pattern can be defined in terms of a family of integer matrices $B(t)$ together with a family of elements $u_{j}(t) \in \mathbb{P}$ satisfying relations (5.4) -(5.5) . In these terms, property (2) above can be rephrased as saying that $b_{i j}\left(t^{\prime}\right)=b_{\sigma(i), \sigma(j)}(t)$ and $u_{j}\left(t^{\prime}\right)=u_{\sigma(j)}(t)$ for all $i, j \in I$.

The following property is an immediate consequence of Proposition 5.4.

Proposition 7.3. Suppose $t$ and $t^{\prime}$ are $\mathcal{M}$-equivalent. For every vertex $t_{1}$ adjacent to $t$, there is a unique vertex $t_{1}^{\prime}$ adjacent to $t^{\prime}$ and equivalent to $t_{1}$. In the notation of Definition [7.1, if $t \frac{\sigma(j)}{\underline{1}} t_{1}$ for some $j \in I$, then $t^{\prime} \stackrel{j}{-} t_{1}^{\prime}$.

Definition 7.4. The exchange graph $\Gamma_{\mathcal{M}}$ associated with a normalized exchange pattern $\mathcal{M}$ has the $\mathcal{M}$-equivalence classes as vertices, joined by an edge if they have adjacent representatives in $\mathbb{T}_{n}$.

As an immediate corollary of Proposition 7.3, we obtain the following.

Corollary 7.5. The exchange graph is n-regular, i.e., every vertex has precisely $n$ edges emanating from it.

Note that passing from $\mathbb{T}_{n}$ to $\Gamma_{\mathcal{M}}$ may result in losing the coloring of edges by elements of the index set $I$. This will happen if a permutation $\sigma$ in Definition 7.1 is nontrivial. (This permutation can be thought of as "discrete monodromy" of our graph.)

Example 7.6. The results in Section 6 (more specifically, Theorem 6.1 and Proposition (6.2) show that, in the rank 2 case, the $\mathcal{M}$-equivalence and the exchange graph can be described as follows. In the infinite case, no two distinct vertices of $\mathbb{T}_{2}$ are 
$\mathcal{M}$-equivalent to each other, so the exchange graph is $\mathbb{T}_{2}$ itself. In the finite case, $t_{m}$ and $t_{m^{\prime}}$ are $\mathcal{M}$-equivalent if and only if $m \equiv m^{\prime} \bmod (h+2)$, so the exchange graph is a cycle of length $h+2$. For example, in the type $A_{2}$ case, we have $h=3$, and the graph is a pentagon. Figure 3 shows the corresponding exchange graph, together with its clusters and exchange relations, written with the help of (2.11) and (6.11). The discrete monodromy is present in this special case, as the variables $y_{1}$ and $y_{2}$ get switched after a full 5 -cycle of exchanges:

$$
\left(\begin{array}{l}
y_{1} \\
y_{2}
\end{array}\right) \rightarrow\left(\begin{array}{l}
y_{3} \\
y_{2}
\end{array}\right) \rightarrow\left(\begin{array}{l}
y_{3} \\
y_{4}
\end{array}\right) \rightarrow\left(\begin{array}{l}
y_{5} \\
y_{4}
\end{array}\right) \rightarrow\left(\begin{array}{l}
y_{5} \\
y_{1}
\end{array}\right) \rightarrow\left(\begin{array}{l}
y_{2} \\
y_{1}
\end{array}\right) .
$$

The corresponding cluster algebra can be realized as the homogeneous coordinate ring of the Grassmannian $G r_{2,5}$; see Example 2.5

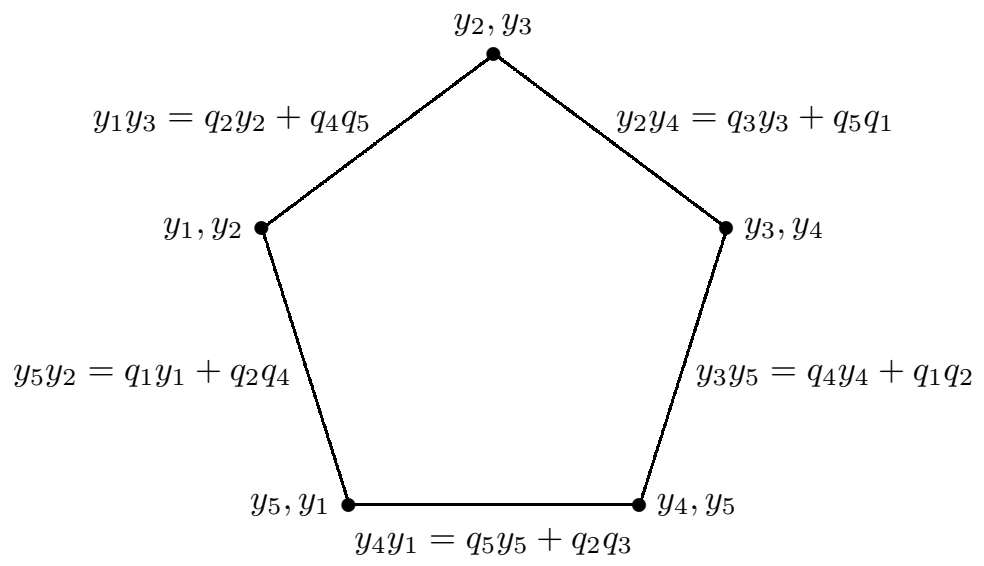

Figure 3 . The exchange graph for a cluster algebra of type $A_{2}$

We see that, in the rank 2 case, the following conditions on a normalized exchange pattern $\mathcal{M}$ are equivalent:

(1) The exchange graph $\Gamma_{\mathcal{M}}$ is finite.

(2) A cluster algebra associated to $\mathcal{M}$ has finitely many distinct cluster variables.

(3) There exists a vertex $t \in \mathbb{T}_{2}$ such that the Cartan counterpart $A(B(t))$ of the matrix $B(t)$ is a Cartan matrix of finite type, i.e., we have $\left|b_{12}(t) b_{21}(t)\right| \leq 3$.

In the sequel to this paper, we shall extend this result to arbitrary rank.

Returning to the general case, we shall now provide some important instances of $\mathcal{M}$-equivalence.

Theorem 7.7. Suppose $\left|b_{i j}(t) b_{j i}(t)\right| \leq 3$ for some vertex $t \in \mathbb{T}_{n}$ and two distinct indices $i$ and $j$. Let $h$ be the Coxeter number of the corresponding rank 2 root system, and let $t^{\prime} \in \mathbb{T}_{n}$ be a vertex joined with $t$ by a path of length $h+2$ whose edge labels alternate between $i$ and $j$. Then $t^{\prime}$ is $\mathcal{M}$-equivalent to $t$, so that the path becomes a cycle of length $h+2$ in the exchange graph.

Proof. For the sake of convenience, assume that $i=1, j=2$; thus, the path joining $t$ and $t^{\prime}$ has the form

$$
t=t_{1} \stackrel{1}{\longrightarrow} t_{2} \stackrel{2}{-} t_{3} \stackrel{1}{-} \cdots \stackrel{\langle h+2\rangle}{\stackrel{h}{2}} t_{h+3}=t^{\prime}
$$


(cf. (2.10) ), where $\langle m\rangle$ as before stands for the element of $\{1,2\}$ congruent to $m$ modulo 2. We abbreviate $b_{1}=-b_{12}\left(t_{1}\right)$ and $b_{2}=b_{21}\left(t_{1}\right)$, and assume (without loss of generality) that $b_{1}$ and $b_{2}$ are either both positive or both equal to 0 . This agrees with the convention (4.5) (also used in Section 6), where $b_{1}$ was denoted by $b$, and $b_{2}$ by $c$. Recall that the value of $h$ is given by Table 1 in Section 6 ; in particular, $h$ is even unless $b_{1}=b_{2}=1$ (the type $A_{2}$ ). We claim that the conditions (1) and (2) in Definition 7.1 hold with the permutation $\sigma$ equal to identity for $h$ even, and equal to the transposition of indices 1 and 2 in the only case when $h$ is odd. Using the restriction operation on exchange patterns (cf. Proposition 2.6), it suffices to treat the case $n=3$, so we can take $I=\{1,2,3\}$.

In view of the exchange property (2.1), the cluster variable $x_{3}\left(t_{m}\right)$ does not depend on $m$. Therefore, verifying condition (1) amounts to checking that $x_{i}\left(t^{\prime}\right)=$ $x_{\sigma(i)}(t)$ for $i \in\{1,2\}$. Again using restriction, we can assume for the purpose of this checking only that $n=2$ and $I=\{1,2\}$, in which case the required property was established in Proposition 6.2

The same argument shows that the only part of condition (2) that does not follow from Proposition 6.2 is the equalities $u_{3}\left(t^{\prime}\right)=u_{3}(t)$ and $b_{i 3}\left(t^{\prime}\right)=b_{\sigma(i), 3}(t)$ for $i \in\{1,2\}$. Let us abbreviate $d_{m}=b_{\langle m\rangle, 3}\left(t_{m}\right)$ and $u_{m+1}=u_{\langle m\rangle}\left(t_{m}\right)$; the latter notation is chosen to be consistent with the notation in the proof of Proposition 6.2 Iterating (5.5) (with $i=3$ ), we obtain

$$
\frac{u_{3}\left(t^{\prime}\right)}{u_{3}(t)}=\prod_{m=1}^{h+2} u_{m+1}^{\max \left(d_{m}, 0\right)}\left(1 \oplus u_{m+1}\right)^{-d_{m}} .
$$

Thus, we need to show that the product on the right-hand side of (7.1) is equal to 1 . To do this, we need some preparation.

Recall that the coefficients $u_{m}$ satisfy the relation (6.9), which in our present notation can be rewritten as

$$
u_{m} u_{m+2}=\left(1 \oplus u_{m+1}\right)^{b_{\langle m\rangle}} .
$$

On the other hand, the matrix mutation rules (4.3) (with $j=3$, applied along the edges $t_{m} \stackrel{\langle m\rangle}{\stackrel{\langle m+1}{\langle m+1}\rangle} t_{m+2}$ ) readily imply the following recurrence for the exponents $d_{m}$ :

$$
d_{m}+d_{m+2}=b_{\langle m\rangle} \max \left(d_{m+1}, 0\right) .
$$

The sequence $\left(u_{m}\right)$ is periodic with period $h+2$, by virtue of Proposition 6.2 Furthermore, the relation (7.3) can be seen, somewhat surprisingly, as a special case of (7.2), for the following version of the tropical semifield: take $\mathbb{Z}$ with the multiplication given by ordinary addition, and the auxiliary addition given by $a \oplus$ $b=\max (a, b)$. This implies in particular that the sequence $\left(d_{m}\right)$ is also periodic with period $h+2$. We also note that the same periodicity holds for the sequence $\left(b_{\langle m\rangle}\right)$ : when $h$ is even, this is clear from the definition of $\langle m\rangle$; and in the only case when $h$ is odd, we have $b_{1}=b_{2}=1$.

To show that the right-hand side of (7.1) is equal to 1 , we first treat the case $b_{1}=b_{2}=0$. Then $h+2=4$, and we have $u_{m+2}=u_{m}^{-1}$ and $d_{m+2}=-d_{m}$ for all $m$. 
It follows that

$$
\begin{aligned}
& \prod_{m=1}^{h+2} u_{m+1}^{\max \left(d_{m}, 0\right)}\left(1 \oplus u_{m+1}\right)^{-d_{m}} \\
& =\prod_{m=1}^{2} u_{m+1}^{\max \left(d_{m}, 0\right)}\left(1 \oplus u_{m+1}\right)^{-d_{m}} u_{m+1}^{-\max \left(-d_{m}, 0\right)}\left(1 \oplus u_{m+1}^{-1}\right)^{d_{m}} \\
& =\prod_{m=1}^{2} u_{m+1}^{d_{m}}\left(1 \oplus u_{m+1}\right)^{-d_{m}}\left(1 \oplus u_{m+1}^{-1}\right)^{d_{m}}=1
\end{aligned}
$$

as desired.

In the remaining case when $b_{1}$ and $b_{2}$ are both positive, we use (7.2) and (7.3) as well as the above-mentioned periodicity, to obtain:

$$
\begin{aligned}
\left(\frac{u_{3}\left(t^{\prime}\right)}{u_{3}(t)}\right)^{b_{1} b_{2}}= & \prod_{m=1}^{h+2} u_{m+1}^{b_{1} b_{2} \max \left(d_{m}, 0\right)}\left(1 \oplus u_{m+1}\right)^{-b_{1} b_{2} d_{m}} \\
= & \prod_{m=1}^{h+2} u_{m+1}^{b_{1} b_{2} \max \left(d_{m}, 0\right)}\left(u_{m} u_{m+2}\right)^{-b_{\langle m+1\rangle} d_{m}} \\
= & u_{1}^{-b_{2} d_{1}} u_{2}^{b_{1}\left(b_{2} \max \left(d_{1}, 0\right)-d_{2}\right)} \\
& \times \prod_{m=1}^{h} u_{m+2}^{-b_{\langle m+1\rangle}\left(d_{m}+d_{m+2}-b_{\langle m\rangle} \max \left(d_{m+1}, 0\right)\right)} \\
& \times u_{h+3}^{b_{\langle h+2\rangle}\left(b_{\langle h+1\rangle} \max \left(d_{h+2}, 0\right)-d_{h+1}\right)} u_{h+4}^{-b_{\langle h+3\rangle} d_{h+2}} \\
= & u_{1}^{-b_{2} d_{1}} u_{2}^{b_{1} d_{0}} u_{h+3}^{b_{\langle h+4} d_{h+3}} u_{h+4}^{-b_{\langle h+3\rangle} d_{h+2}} \\
= & u_{1}^{\left(b_{\langle h+4\rangle}-b_{2}\right) d_{1}} u_{2}^{\left(b_{1}-b_{\langle h+3\rangle}\right) d_{0}}=1 .
\end{aligned}
$$

To conclude that $u_{3}\left(t^{\prime}\right)=u_{3}(t)$, just recall that the coefficient group $\mathbb{P}$ is assumed to be torsion-free.

To complete the proof of the theorem, it remains to show that $b_{i 3}\left(t^{\prime}\right)=b_{\sigma(i), 3}(t)$ for $i \in\{1,2\}$. But this is equivalent to saying that the sequence $\left(d_{m}\right)$ is periodic with period $h+2$, which is already proven.

We conjecture that the $\mathcal{M}$-equivalence relation is generated by its instances described in Theorem 7.7

Example 7.8. Let $\mathcal{M}(B)$ be an exchange pattern of geometric type associated with the skew-symmetric matrix

$$
\tilde{B}=B=\left[\begin{array}{ccc}
0 & 1 & 1 \\
-1 & 0 & 1 \\
-1 & -1 & 0
\end{array}\right] \text {, }
$$

as prescribed by Corollary [5.9] Thus, the corresponding cluster algebra is of rank 3, and all the coefficients $p_{j}(t)$ are equal to 1 . The exchange graph for this pattern is a "two-layer brick wall" shown in Figure 4 In this figure, distinct cluster variables are associated with regions: there are variables $y_{m}(m \in \mathbb{Z})$ associated with bounded regions ("bricks"), and two more variables $w$ and $z$ associated with the two unbounded regions. The cluster at any vertex $t$ consists of the three cluster 
variables associated to the three regions adjacent to $t$. The binomial exchange relations are as follows: for $m \in \mathbb{Z}$, we have

$$
\begin{aligned}
& w y_{2 m}=y_{2 m-1}+y_{2 m+1}, \\
& y_{2 m-1} y_{2 m+3}=y_{2 m+1}^{2}+w, \\
& y_{m} y_{m+3}=y_{m+1} y_{m+2}+1, \\
& y_{2 m-2} y_{2 m+2}=y_{2 m}^{2}+z, \\
& y_{2 m-1} z=y_{2 m-2}+y_{2 m} .
\end{aligned}
$$

To see all this, we first show that the graph in Figure 4 is a cover of the exchange graph $\Gamma_{\mathcal{M}}$. Pick an initial vertex $t_{0} \in \Gamma_{\mathcal{M}}$ with the matrix $B\left(t_{0}\right)=B$ given by (17.4) (abusing notation, we will use the same symbol for a vertex in $\mathbb{T}_{3}$ and its image in $\Gamma_{\mathcal{M}}$ ). Denote the cluster variables at $t_{0}$ by $y_{1}, y_{2}$, and $y_{3}$, so that their order agrees with that of rows and columns of $B$. Since every principal $2 \times 2$ submatrix of $B$ is of type $A_{2}$, Theorem 7.7 provides that $t_{0}$ is a common vertex of three 5 -cycles in $\Gamma_{\mathcal{M}}$. These cycles are depicted in Figure 4 as perimeters of the three bricks surrounding $t_{0}$. The variable $y_{i}$ inside each brick indicates that this brick corresponds to the rank 2 exchange pattern obtained from $\mathcal{M}$ via restriction from $I=[3]$ to $[3] \backslash\{i\}$; equivalently, this means that $y_{i}$ appears in every cluster on the perimeter of the brick. Now we move from $t_{0}$ to an adjacent vertex $t_{1}$ with the cluster $\left\{y_{2}, y_{3}, y_{4}\right\}$. According to Proposition 4.3 .

$$
B\left(t_{1}\right)=\mu_{1}(B)=\left[\begin{array}{ccc}
0 & -1 & -1 \\
1 & 0 & 1 \\
1 & -1 & 0
\end{array}\right] .
$$

This matrix differs from $B$ by a simultaneous cyclic permutation of rows and columns. Therefore, we can apply the same construction to $t_{1}$, obtaining the three bricks surrounding this vertex. Continuing in the same way, we produce the entire graph in Figure 4 Since this graph is 3-regular, we have covered all the vertices and edges of the exchange graph.

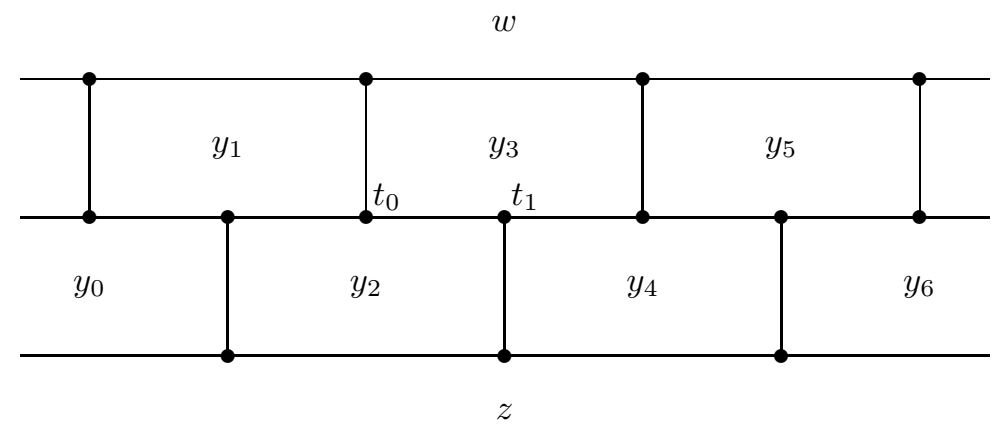

Figure 4. The two-layer brick wall

For every vertex $t$ on the median of the wall, the matrix $B(t)$ is obtained from $B$ by a simultaneous permutation of rows and columns. Now take a vertex on the 
outer boundary, say the vertex $t_{2}$ in Figure 5 Then

$$
B\left(t_{2}\right)=\mu_{3}\left(B\left(t_{1}\right)\right)=\left[\begin{array}{ccc}
0 & -2 & 1 \\
2 & 0 & -1 \\
-1 & 1 & 0
\end{array}\right] .
$$

Again, for every other vertex on the outer boundary, the corresponding matrix is obtained from $B\left(t_{2}\right)$ by a simultaneous permutation of rows and columns. Substituting the matrices $B(t)$ into (4.2), we generate all the exchange relations (17.5) (cf. Figure [5).

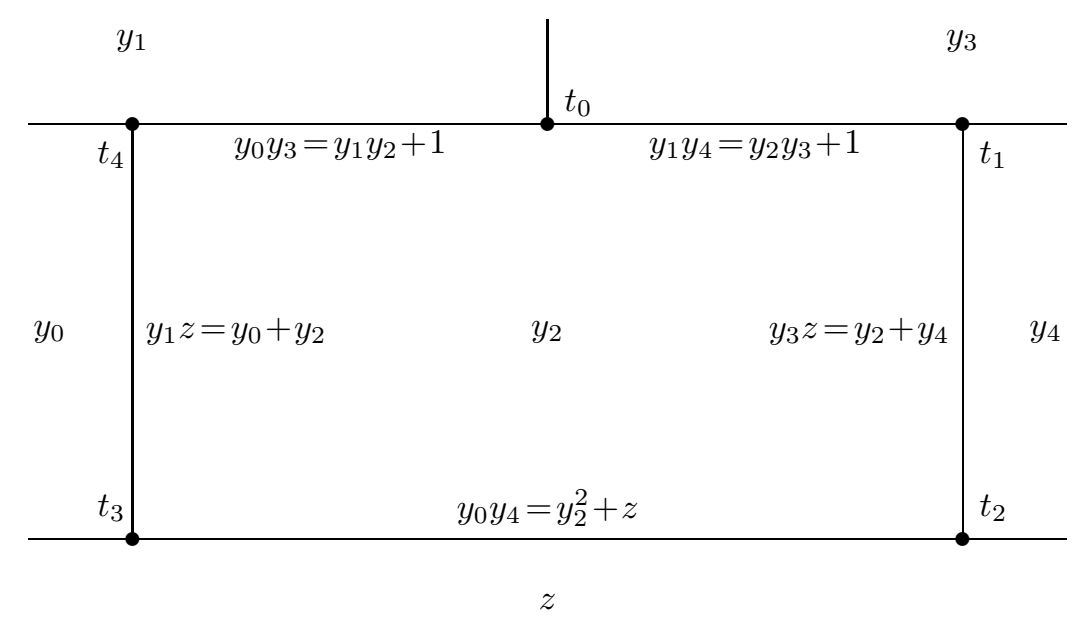

Figure 5. Close-up of a brick

To prove that our brick wall is indeed the exchange graph, it remains to show that all the cluster variables $y_{m}, w$, and $z$ are distinct; recall that we view them as elements of the ambient field $\mathcal{F}$. Following the methodology of Theorem 6.1 it suffices to show that all of them have different denominators in the Laurent expansion in terms of the initial cluster $\left\{y_{1}, y_{2}, y_{3}\right\}$. We write the denominator of a cluster variable $y$ as $y_{1}^{d_{1}(y)} y_{2}^{d_{2}(y)} y_{3}^{d_{3}(y)}$ (cf. (6.2)), and encode it by a vector $\delta(y)=\left(d_{1}(y), d_{2}(y), d_{3}(y)\right) \in \mathbb{Z}^{3}$. In particular, we have

$$
\delta\left(y_{1}\right)=(-1,0,0), \delta\left(y_{2}\right)=(0,-1,0), \delta\left(y_{3}\right)=(0,0,-1) .
$$

A moment's reflection shows that the vectors $\delta\left(y_{m}\right), \delta(w)$, and $\delta(z)$ satisfy the "tropical version" of each of the exchange relations (7.5) (with multiplication replaced by vector addition, and addition replaced by component-wise maximum), as long as the two vectors on the right-hand side are different. For example, the first equation in 7.5 will take the form

$$
\delta(w)+\delta\left(y_{2 m}\right)=\max \left(\delta\left(y_{2 m-1}\right), \delta\left(y_{2 m+1}\right)\right),
$$

provided $\delta\left(y_{2 m-1}\right) \neq \delta\left(y_{2 m+1}\right)$; here max stands for component-wise maximum. All $\delta(y)$ 's are uniquely determined from (17.6) by recursive application of these 
conditions. As a result, we obtain, for every $m \geq 0$ :

$$
\begin{array}{lll}
\delta\left(y_{1-2 m}\right)=(m-1, m, m), & & \\
\delta\left(y_{-2 m}\right)=(m, m, m+1), & & \delta\left(y_{2 m+3}\right)=(m, m, m-1), \\
& \delta\left(y_{2 m+4}\right)=(m+1, m, m), \\
& \delta(z)=(1,0,1) &
\end{array}
$$

(cf. Figure 6, where each vector $\delta(y)$ is shown within the corresponding region).

$w$

$(0,1,0)$

\begin{tabular}{c|c|c|c|c|c|c|c|}
\hline & $y_{-3}$ & $y_{-1}$ & $y_{1}$ & $y_{3}$ & $y_{5}$ & $y_{7}$ \\
\multicolumn{1}{|c|}{$(1,2,2)$} & $(0,1,1)$ & $(-1,0,0)$ & $(0,0,-1)$ & $(1,1,0)$ & $(2,2,1)$ \\
\hline$y_{-4}$ & $y_{-2}$ & $y_{0}$ & $y_{2}$ & $y_{4}$ & $y_{6}$ & $y_{8}$ \\
$(2,2,3)$ & $(1,1,2)$ & $(0,0,1)$ & $(0,-1,0)$ & $(1,0,0)$ & $(2,1,1)$ & $(3,2,2)$ \\
\hline
\end{tabular}

$(1,0,1)$

Figure 6 . The brick wall denominators

\section{ACKNOWLEDGMENTS}

This work began in May 2000 when the authors got together as participants in the program "Representation Theory-2000," organized by Victor Kac and Alexander A. Kirillov at the Erwin Schrödinger International Institute for Mathematical Physics in Vienna, Austria. We thank the organizers for inviting us, and we are grateful to Peter Michor and the staff of the Institute for creating ideal working conditions. The paper was finished at the Isaac Newton Institute for Mathematical Sciences in Cambridge, UK, whose support is gratefully acknowledged.

It was during our stay in Vienna that we learned of the terminal illness of Sergei Kerov, an outstanding mathematician and a good friend of ours. He passed away on July 30, 2000. We dedicate this paper to his memory.

\section{REFERENCES}

[1] A. Berenstein, S. Fomin and A. Zelevinsky, Parametrizations of canonical bases and totally positive matrices, Adv. Math. 122 (1996), 49-149. MR 98j:17008

[2] A. Berenstein and A. Zelevinsky, String bases for quantum groups of type $A_{r}$, in I. M. Gelfand Seminar, 51-89, Adv. Soviet Math. 16, Part 1, Amer. Math. Soc., Providence, RI, 1993. MR 94g:17019

[3] A. Berenstein and A. Zelevinsky, Total positivity in Schubert varieties, Comment. Math. Helv. 72 (1997), 128-166. MR 99g:14064

[4] A. Berenstein and A. Zelevinsky, Tensor product multiplicities, canonical bases and totally positive varieties, Invent. Math. 143 (2001), 77-128. CMP 2001:06

[5] S. Fomin and A. Zelevinsky, Double Bruhat cells and total positivity, J. Amer. Math. Soc. 12 (1999), 335-380. MR 2001f:20097

[6] S. Fomin and A. Zelevinsky, Total positivity: tests and parametrizations, Math. Intelligencer 22 (2000), no. 1, 23-33. MR 2001b:15030

[7] S. Fomin and A. Zelevinsky, Totally nonnegative and oscillatory elements in semisimple groups, Proc. Amer. Math. Soc. 128 (2000), 3749-3759. MR 2001b:22014

[8] S. Fomin and A. Zelevinsky, The Laurent phenomenon, to appear in Adv. in Applied Math. 
[9] I. M. Gelfand and A. Zelevinsky, Canonical basis in irreducible representations of $g l_{3}$ and its applications, in: Group theoretical methods in physics, Vol. II (Jurmala, 1985), 127-146, VNU Sci. Press, Utrecht, 1986. MR 89a:17010

[10] V. Kac, Infinite dimensional Lie algebras, 3rd edition, Cambridge University Press, 1990. MR 92k:17038

[11] J. Kung and G.-C. Rota, The invariant theory of binary forms, Bull. Amer. Math. Soc. (N.S.) 10 (1984), 27-85. MR 85g:05002

[12] B. Leclerc and A. Zelevinsky, Quasicommuting families of quantum Plucker coordinates, Amer. Math. Soc. Transl. (2), Vol. 181, Kirillov's Seminar on Representation Theory, 85108, Amer. Math. Soc., Providence, RI, 1998. MR 99g:14066

[13] G. Lusztig, Canonical bases arising from quantized enveloping algebras, J. Amer. Math. Soc. 3 (1990), 447-498. MR 90m:17023

[14] G. Lusztig, Introduction to quantum groups, Progress in Mathematics 110, Birkhäuser, 1993. MR 94m:17016

[15] G. Lusztig, Total positivity in reductive groups, in: Lie theory and geometry: in honor of Bertram Kostant, Progress in Mathematics 123, Birkhäuser, 1994. MR 96m:20071

[16] V. Retakh and A. Zelevinsky, The base affine space and canonical bases in irreducible representations of the group Sp(4), Soviet Math. Dokl. 37 (1988), no. 3, 618-622. MR 89g:22025

[17] B. Shapiro, M. Shapiro, A. Vainshtein and A. Zelevinsky, Simply-laced Coxeter groups and groups generated by symplectic transvections, Michigan Math. J. 48 (2000), 531-551. MR 2001g:20050

[18] B. Sturmfels, Algorithms in invariant theory. Texts and Monographs in Symbolic Computation. Springer-Verlag, Vienna, 1993. MR 94m:13004

[19] Al. B. Zamolodchikov, On the thermodynamic Bethe ansatz equations for reflectionless $A D E$ scattering theories, Phys. Lett. B 253 (1991), 391-394. MR 92a:81196

[20] A. Zelevinsky, Connected components of real double Bruhat cells, Intern. Math. Res. Notices 2000, No. 21, 1131-1153. MR 2001k:14094

Department of Mathematics, University of Michigan, Ann Arbor, Michigan 48109

E-mail address: fomin@umich.edu

Department of Mathematics, Northeastern University, Boston, Massachusetts 02115

E-mail address: andrei@neu.edu 